\title{
Endpoint estimates for commutators of singular integrals related to Schrödinger operators
}

\author{
Luong Dang Ky
}

\begin{abstract}
Let $L=-\Delta+V$ be a Schrödinger operator on $\mathbb{R}^{d}, d \geq 3$, where $V$ is a nonnegative potential, $V \neq 0$, and belongs to the reverse Hölder class $R H_{d / 2}$. In this paper, we study the commutators $[b, T]$ for $T$ in a class $\mathcal{K}_{L}$ of sublinear operators containing the fundamental operators in harmonic analysis related to $L$. More precisely, when $T \in \mathcal{K}_{L}$, we prove that there exists a bounded subbilinear operator $\mathfrak{R}=\mathfrak{R}_{T}: H_{L}^{1}\left(\mathbb{R}^{d}\right) \times \mathrm{BMO}\left(\mathbb{R}^{d}\right) \rightarrow$ $L^{1}\left(\mathbb{R}^{d}\right)$ such that

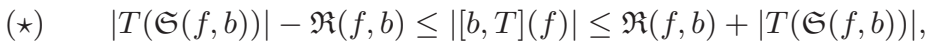

where $\mathfrak{S}$ is a bounded bilinear operator from $H_{L}^{1}\left(\mathbb{R}^{d}\right) \times \mathrm{BMO}\left(\mathbb{R}^{d}\right)$ into $L^{1}\left(\mathbb{R}^{d}\right)$ which does not depend on $T$. The subbilinear decomposition $(\star)$ allows us to explain why commutators with the fundamental operators are of weak type $\left(H_{L}^{1}, L^{1}\right)$, and when a commutator $[b, T]$ is of strong type $\left(H_{L}^{1}, L^{1}\right)$.

Also, we discuss the $H_{L}^{1}$-estimates for commutators of the Riesz transforms associated with the Schrödinger operator $L$.
\end{abstract}

\section{Introduction}

Given a function $b$ locally integrable on $\mathbb{R}^{d}$, and a (classical) Calderón-Zygmund operator $T$, we consider the linear commutator $[b, T]$ defined for smooth, compactly supported functions $f$ by

$$
[b, T](f)=b T(f)-T(b f) .
$$

A classical result of Coifman, Rochberg and Weiss (see [12]), states that the commutator $[b, T]$ is continuous on $L^{p}\left(\mathbb{R}^{d}\right)$ for $1<p<\infty$, when $b \in \mathrm{BMO}\left(\mathbb{R}^{d}\right)$. Unlike the theory of (classical) Calderón-Zygmund operators, the proof of this result does not rely on a weak type $(1,1)$ estimate for $[b, T]$. Instead, an endpoint theory was

Mathematics Subject Classification (2010): Primary 42B35, 35J10; Secondary 42B20.

Keywords: Schrödinger operators, commutators, Hardy spaces, Calderón-Zygmund operators, Riesz transforms, BMO, atoms. 
provided for this operator, see for example [37], [38]. A general overview about these facts can be found for instance in [28].

Let $L=-\Delta+V$ be a Schrödinger operator on $\mathbb{R}^{d}, d \geq 3$, where $V$ is a nonnegative potential, $V \neq 0$, and belongs to the reverse Hölder class $R H_{d / 2}$. We recall that a nonnegative locally integrable function $V$ belongs to the reverse Hölder class $R H_{q}, 1<q<\infty$, if there exists $C>0$ such that

$$
\left(\frac{1}{|B|} \int_{B}(V(x))^{q} d x\right)^{1 / q} \leq \frac{C}{|B|} \int_{B} V(x) d x
$$

holds for every balls $B$ in $\mathbb{R}^{d}$. In [16], Dziubański and Zienkiewicz introduced the Hardy space $H_{L}^{1}\left(\mathbb{R}^{d}\right)$ as the set of functions $f \in L^{1}\left(\mathbb{R}^{d}\right)$ such that $\|f\|_{H_{L}^{1}}:=$ $\left\|\mathcal{M}_{L} f\right\|_{L^{1}}<\infty$, where $\mathcal{M}_{L} f(x):=\sup _{t>0}\left|e^{-t L} f(x)\right|$. There, they characterized $H_{L}^{1}\left(\mathbb{R}^{d}\right)$ in terms of atomic decomposition and in terms of the Riesz transforms associated with $L, R_{j}=\partial_{x_{j}} L^{-1 / 2}, j=1, \ldots, d$. In the recent years, there is an increasing interest on the study of commutators of singular integral operators related to Schrödinger operators, see for example [7], [10], [21], [32], [43], [44], [45].

In the present paper, we consider commutators of singular integral operators $T$ related to the Schrödinger operator $L$. Here $T$ is in the class $\mathcal{K}_{L}$ of all sublinear operators $T$, bounded from $H_{L}^{1}\left(\mathbb{R}^{d}\right)$ into $L^{1}\left(\mathbb{R}^{d}\right)$ and satisfying for any $b \in \operatorname{BMO}\left(\mathbb{R}^{d}\right)$ and $a$ a generalized atom related to the ball $B$ (see Definition 2.3), we have

$$
\left\|\left(b-b_{B}\right) T a\right\|_{L^{1}} \leq C\|b\|_{\mathrm{BMO}}
$$

where $b_{B}$ denotes the average of $b$ on $B$ and $C>0$ is a constant independent of $b$ and $a$. The class $\mathcal{K}_{L}$ contains the fundamental operators (we refer the reader to [28] for the classical case $L=-\Delta$ ) related to the Schrödinger operator $L$ : the Riesz transforms $R_{j}, L$-Calderón-Zygmund operators (so-called SchrödingerCalderón-Zygmund operators), $L$-maximal operators, $L$-square operators, etc. (see Section 4). It should be pointed out that, by the work of Shen [39] and Definition 2.9 (see Remark 2.10), one only can conclude that the Riesz transforms $R_{j}$ are Schrödinger-Calderón-Zygmund operators whenever $V \in R H_{d}$. In this work, we consider all potentials $V$ which belong to the reverse Hölder class $R H_{d / 2}$.

Although Schrödinger-Calderón-Zygmund operators map $H_{L}^{1}\left(\mathbb{R}^{d}\right)$ into $L^{1}\left(\mathbb{R}^{d}\right)$ (see Proposition 4.1), it was observed in [32], [48] that, when $b \in \operatorname{BMO}\left(\mathbb{R}^{d}\right)$, the commutators $\left[b, R_{j}\right]$ do not map, in general, $H_{L}^{1}\left(\mathbb{R}^{d}\right)$ into $L^{1}\left(\mathbb{R}^{d}\right)$. In the classical setting, it was derived by M. Paluszyński [35] that the commutator of the Hilbert transform $[b, H]$ does not map, in general, $H^{1}(\mathbb{R})$ into $L^{1}(\mathbb{R})$. After, C. Pérez showed in [37] that if $H^{1}\left(\mathbb{R}^{d}\right)$ is replaced by a suitable atomic subspace $H_{b}^{1}\left(\mathbb{R}^{d}\right)$ then commutators of the classical Calderón-Zygmund operators are continuous from $H_{b}^{1}\left(\mathbb{R}^{d}\right)$ into $L^{1}\left(\mathbb{R}^{d}\right)$. Recall that (see [37]) a function $a$ is a $b$-atom if

i) supp $a \subset Q$ for some cube $Q$,

ii) $\|a\|_{L^{\infty}} \leq|Q|^{-1}$,

iii) $\int_{\mathbb{R}^{d}} a(x) d x=\int_{\mathbb{R}^{d}} a(x) b(x) d x=0$. 
The space $H_{b}^{1}\left(\mathbb{R}^{d}\right)$ consists of the subspace of $L^{1}\left(\mathbb{R}^{d}\right)$ of functions $f$ which can be written as $f=\sum_{j=1}^{\infty} \lambda_{j} a_{j}$ where $a_{j}$ are $b$-atoms, and $\lambda_{j}$ are complex numbers with $\sum_{j=1}^{\infty}\left|\lambda_{j}\right|<\infty$. Thus, when $b \in \mathrm{BMO}\left(\mathbb{R}^{d}\right)$, it is natural to ask for subspaces of $H_{L}^{1}\left(\mathbb{R}^{d}\right)$ such that all commutators of Schrödinger-Calderón-Zygmund operators and the Riesz transforms map continuously these spaces into $L^{1}\left(\mathbb{R}^{d}\right)$.

In this paper, we are interested in the following two questions.

Question 1. For $b \in \mathrm{BMO}\left(\mathbb{R}^{d}\right)$. Find the largest subspace $\mathcal{H}_{L, b}^{1}\left(\mathbb{R}^{d}\right)$ of $H_{L}^{1}\left(\mathbb{R}^{d}\right)$ such that all commutators of Schrödinger-Calderón-Zygmund operators and the Riesz transforms are bounded from $\mathcal{H}_{L, b}^{1}\left(\mathbb{R}^{d}\right)$ into $L^{1}\left(\mathbb{R}^{d}\right)$.

Question 2. Characterize functions $b$ in $\mathrm{BMO}\left(\mathbb{R}^{d}\right)$ so that $\mathcal{H}_{L, b}^{1}\left(\mathbb{R}^{d}\right) \equiv H_{L}^{1}\left(\mathbb{R}^{d}\right)$.

Let $X$ be a Banach space. We say that an operator $T: X \rightarrow L^{1}\left(\mathbb{R}^{d}\right)$ is a sublinear operator if for all $f, g \in X$ and $\alpha, \beta \in \mathbb{C}$, we have

$$
|T(\alpha f+\beta g)(x)| \leq|\alpha||T f(x)|+|\beta||T g(x)| \text {. }
$$

Obviously, a linear operator $T: X \rightarrow L^{1}\left(\mathbb{R}^{d}\right)$ is a sublinear operator. We also say that an operator $\mathfrak{T}: H_{L}^{1}\left(\mathbb{R}^{d}\right) \times \mathrm{BMO}\left(\mathbb{R}^{d}\right) \rightarrow L^{1}\left(\mathbb{R}^{d}\right)$ is a subbilinear operator if for every $(f, g) \in H_{L}^{1}\left(\mathbb{R}^{d}\right) \times \operatorname{BMO}\left(\mathbb{R}^{d}\right)$, the operators $\mathfrak{T}(f, \cdot): \operatorname{BMO}\left(\mathbb{R}^{d}\right) \rightarrow L^{1}\left(\mathbb{R}^{d}\right)$ and $\mathfrak{T}(\cdot, g): H_{L}^{1}\left(\mathbb{R}^{d}\right) \rightarrow L^{1}\left(\mathbb{R}^{d}\right)$ are sublinear operators.

To answer Questions 1 and 2, we study commutators of sublinear operators in $\mathcal{K}_{L}$. More precisely, when $T \in \mathcal{K}_{L}$ is a sublinear operator, we prove (see Theorem 3.1) that there exists a bounded subbilinear operator $\mathfrak{R}=\mathfrak{R}_{T}: H_{L}^{1}\left(\mathbb{R}^{d}\right) \times$ $\operatorname{BMO}\left(\mathbb{R}^{d}\right) \rightarrow L^{1}\left(\mathbb{R}^{d}\right)$ so that for all $(f, b) \in H_{L}^{1}\left(\mathbb{R}^{d}\right) \times \operatorname{BMO}\left(\mathbb{R}^{d}\right)$,

$$
|T(\mathfrak{S}(f, b))|-\mathfrak{R}(f, b) \leq|[b, T](f)| \leq \mathfrak{R}(f, b)+|T(\mathfrak{S}(f, b))|,
$$

where $\mathfrak{S}$ is a bounded bilinear operator from $H_{L}^{1}\left(\mathbb{R}^{d}\right) \times \mathrm{BMO}\left(\mathbb{R}^{d}\right)$ into $L^{1}\left(\mathbb{R}^{d}\right)$ which does not depend on $T$ (see Proposition 5.6). When $T \in \mathcal{K}_{L}$ is a linear operator, we prove (see Theorem 3.4) that there exists a bounded bilinear operator $\mathfrak{R}=$ $\mathfrak{R}_{T}: H_{L}^{1}\left(\mathbb{R}^{d}\right) \times \operatorname{BMO}\left(\mathbb{R}^{d}\right) \rightarrow L^{1}\left(\mathbb{R}^{d}\right)$ such that for all $(f, b) \in H_{L}^{1}\left(\mathbb{R}^{d}\right) \times \operatorname{BMO}\left(\mathbb{R}^{d}\right)$,

$$
[b, T](f)=\mathfrak{R}(f, b)+T(\mathfrak{S}(f, b)) .
$$

The decompositions (1.1) and (1.2) give a general overview and explains why almost commutators of the fundamental operators are of weak type $\left(H_{L}^{1}, L^{1}\right)$, and when a commutator $[b, T]$ is of strong type $\left(H_{L}^{1}, L^{1}\right)$.

Let $b$ be a function in $\operatorname{BMO}\left(\mathbb{R}^{d}\right)$. We assume that $b$ non-constant, otherwise $[b, T]=0$. We define the space $\mathcal{H}_{L, b}^{1}\left(\mathbb{R}^{d}\right)$ as the set of all $f$ in $H_{L}^{1}\left(\mathbb{R}^{d}\right)$ such that $\left[b, \mathcal{M}_{L}\right](f)(x)=\mathcal{M}_{L}(b(x) f(\cdot)-b(\cdot) f(\cdot))(x)$ belongs to $L^{1}\left(\mathbb{R}^{d}\right)$, and the norm on $\mathcal{H}_{L, b}^{1}\left(\mathbb{R}^{d}\right)$ is defined by $\|f\|_{\mathcal{H}_{L, b}^{1}}=\|f\|_{H_{L}^{1}}\|b\|_{\mathrm{BMO}}+\left\|\left[b, \mathcal{M}_{L}\right](f)\right\|_{L^{1}}$. Then, using the subbilinear decomposition (1.1), we prove that all commutators of Schrödinger-Calderón-Zygmund operators and the Riesz transforms are bounded from $\mathcal{H}_{L, b}^{1}\left(\mathbb{R}^{d}\right)$ into $L^{1}\left(\mathbb{R}^{d}\right)$. Furthermore, $\mathcal{H}_{L, b}^{1}\left(\mathbb{R}^{d}\right)$ is the largest space having 
this property, and $\mathcal{H}_{L, b}^{1}\left(\mathbb{R}^{d}\right) \equiv H_{L}^{1}\left(\mathbb{R}^{d}\right)$ if and only if $b \in \mathrm{BMO}_{L}^{\log }\left(\mathbb{R}^{d}\right)$ (see Theorem 7.5), that is,

$$
\|b\|_{\mathrm{BMO}_{L}^{\log }}=\sup _{B(x, r)}\left(\log \left(e+\frac{\rho(x)}{r}\right) \frac{1}{|B(x, r)|} \int_{B(x, r)}\left|b(y)-b_{B(x, r)}\right| d y\right)<\infty,
$$

where $\rho(x)=\sup \left\{r>0: \frac{1}{r^{d-2}} \int_{B(x, r)} V(y) d y \leq 1\right\}$. This space $\mathrm{BMO}_{L}^{\log }\left(\mathbb{R}^{d}\right)$ arises naturally in the characterization of pointwise multipliers for $\mathrm{BMO}_{L}\left(\mathbb{R}^{d}\right)$, the dual space of $H_{L}^{1}\left(\mathbb{R}^{d}\right)$, see [3], [33].

The above answers Questions 1 and 2. As another interesting application of the subbilinear decomposition (1.1), we find subspaces of $H_{L}^{1}\left(\mathbb{R}^{d}\right)$ which do not depend on $b \in \operatorname{BMO}\left(\mathbb{R}^{d}\right)$ and $T \in \mathcal{K}_{L}$, such that $[b, T]$ maps continuously these spaces into $L^{1}\left(\mathbb{R}^{d}\right)$ (see Section 7 ). For instance, when $L=-\Delta+1$, Theorem 7.10 state that for every $b \in \mathrm{BMO}\left(\mathbb{R}^{d}\right)$ and $T \in \mathcal{K}_{L}$, the commutator $[b, T]$ is bounded from $H_{L}^{1,1}\left(\mathbb{R}^{d}\right)$ into $L^{1}\left(\mathbb{R}^{d}\right)$. Here $H_{L}^{1,1}\left(\mathbb{R}^{d}\right)$ is the (inhomogeneous) Hardy-Sobolev space considered by Hofmann, Mayboroda and McIntosh in [23], defined as the set of functions $f$ in $H_{L}^{1}\left(\mathbb{R}^{d}\right)$ such that $\partial_{x_{1}} f, \ldots, \partial_{x_{d}} f \in H_{L}^{1}\left(\mathbb{R}^{d}\right)$ with the norm

$$
\|f\|_{H_{L}^{1,1}}=\|f\|_{H_{L}^{1}}+\sum_{j=1}^{d}\left\|\partial_{x_{j}} f\right\|_{H_{L}^{1}} .
$$

Recently, similarly to the classical result of Coifman-Rochberg-Weiss, Gou et al. proved in $[21]$ that the commutators $\left[b, R_{j}\right]$ are bounded on $L^{p}\left(\mathbb{R}^{d}\right)$ whenever $b \in \operatorname{BMO}\left(\mathbb{R}^{d}\right)$ and $1<p<\frac{d q}{d-q}$ where $V \in R H_{q}$ for some $d / 2<q<d$. Later, in [7], Bongioanni et al. generalized this result by showing that the space $\mathrm{BMO}\left(\mathbb{R}^{d}\right)$ can be replaced by a larger space $\mathrm{BMO}_{L, \infty}\left(\mathbb{R}^{d}\right)=\cup_{\theta \geq 0} \mathrm{BMO}_{L, \theta}\left(\mathbb{R}^{d}\right)$, where $\mathrm{BMO}_{L, \theta}\left(\mathbb{R}^{d}\right)$ is the space of locally integrable functions $f$ satisfying

$$
\|f\|_{\mathrm{BMO}_{L, \theta}}=\sup _{B(x, r)}\left(\frac{1}{\left(1+\frac{r}{\rho(x)}\right)^{\theta}} \frac{1}{|B(x, r)|} \int_{B(x, r)}\left|f(y)-f_{B(x, r)}\right| d y\right)<\infty .
$$

Let $R_{j}^{*}$ be the adjoint operators of $R_{j}$. Bongioanni et al. established in [6] that the operators $R_{j}^{*}$ are bounded on $\mathrm{BMO}_{L}\left(\mathbb{R}^{d}\right)$, and thus from $L^{\infty}\left(\mathbb{R}^{d}\right)$ into $\mathrm{BMO}_{L}\left(\mathbb{R}^{d}\right)$. Therefore, it is natural to ask for a class of functions $b$ so that the commutators $\left[b, R_{j}^{*}\right]$ map continuously $L^{\infty}\left(\mathbb{R}^{d}\right)$ into $\mathrm{BMO}_{L}\left(\mathbb{R}^{d}\right)$. In $[7]$, the authors found such a class of functions. More precisely, they proved in [7] that the commutators $\left[b, R_{j}^{*}\right]$ map continuously $L^{\infty}\left(\mathbb{R}^{d}\right)$ into $\mathrm{BMO}_{L}\left(\mathbb{R}^{d}\right)$ whenever $b \in$ $\mathrm{BMO}_{L, \infty}^{\log }\left(\mathbb{R}^{d}\right)=\cup_{\theta \geq 0} \mathrm{BMO}_{L, \theta}^{\log }\left(\mathbb{R}^{d}\right)$. Here $\mathrm{BMO}_{L, \theta}^{\log }\left(\mathbb{R}^{d}\right)$ is the space of functions $f \in L_{\mathrm{loc}}^{1}\left(\mathbb{R}^{d}\right)$ such that

$$
\|f\|_{\mathrm{BMO}_{L, \theta}^{\log }}=\sup _{B(x, r)}\left(\frac{\log \left(e+\frac{\rho(x)}{r}\right)}{\left(1+\frac{r}{\rho(x)}\right)^{\theta}} \frac{1}{|B(x, r)|} \int_{B(x, r)}\left|f(y)-f_{B(x, r)}\right| d y\right)<\infty .
$$

A natural question arises: can one replace the space $L^{\infty}\left(\mathbb{R}^{d}\right)$ by $\operatorname{BMO}_{L}\left(\mathbb{R}^{d}\right)$ ? 
Question 3. Are the commutators $\left[b, R_{j}^{*}\right], j=1, \ldots, d$, bounded on $\mathrm{BMO}_{L}\left(\mathbb{R}^{d}\right)$ whenever $b \in \mathrm{BMO}_{L, \infty}^{\log }\left(\mathbb{R}^{d}\right)$ ?

Motivated by this question, we study the $H_{L}^{1}$-estimates for commutators of the Riesz transforms. More precisely, given $b \in \mathrm{BMO}_{L, \infty}\left(\mathbb{R}^{d}\right)$, we prove that the commutators $\left[b, R_{j}\right]$ are bounded on $H_{L}^{1}\left(\mathbb{R}^{d}\right)$ if and only if $b$ belongs to $\mathrm{BMO}_{L, \infty}^{\log }\left(\mathbb{R}^{d}\right)$ (see Theorem 3.6). Furthermore, if $b \in \mathrm{BMO}_{L, \theta}^{\log }\left(\mathbb{R}^{d}\right)$ for some $\theta \geq 0$, then there exists a constant $C>1$, independent of $b$, such that

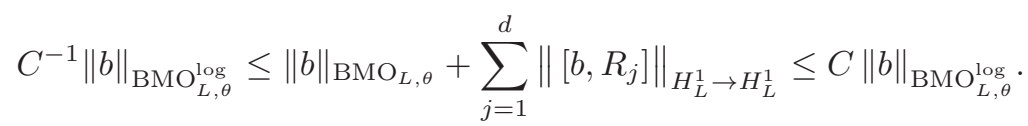

As a consequence, we get the positive answer for Question 3.

Now, an open question is the following:

Open question. Find the set of all functions $b$ such that the commutators $\left[b, R_{j}\right]$, $j=1, \ldots, d$, are bounded on $H_{L}^{1}\left(\mathbb{R}^{d}\right)$.

Let us emphasize the three main purposes of this paper. First, we prove the two decomposition theorems: the subbilinear decomposition (1.1) and the bilinear decomposition (1.2). Second, we characterize functions $b$ in $\mathrm{BMO}_{L, \infty}\left(\mathbb{R}^{d}\right)$ so that the commutators of the Riesz transforms are bounded on $H_{L}^{1}\left(\mathbb{R}^{d}\right)$, which answers Question 3. Finally, we find the largest subspace $\mathcal{H}_{L, b}^{1}\left(\mathbb{R}^{d}\right)$ of $H_{L}^{1}\left(\mathbb{R}^{d}\right)$ such that all commutators of Schrödinger-Calderón-Zygmund operators and the Riesz transforms are bounded from $\mathcal{H}_{L, b}^{1}\left(\mathbb{R}^{d}\right)$ into $L^{1}\left(\mathbb{R}^{d}\right)$. Besides, we find also the characterization of functions $b \in \operatorname{BMO}\left(\mathbb{R}^{d}\right)$ so that $\mathcal{H}_{L, b}^{1}\left(\mathbb{R}^{d}\right) \equiv H_{L}^{1}\left(\mathbb{R}^{d}\right)$, which answer Questions 1 and 2. Especially, we show that there exist subspaces of $H_{L}^{1}\left(\mathbb{R}^{d}\right)$ which do not depend on $b \in \mathrm{BMO}\left(\mathbb{R}^{d}\right)$ and $T \in \mathcal{K}_{L}$, such that $[b, T]$ maps continuously these spaces into $L^{1}\left(\mathbb{R}^{d}\right)$, see Section 7 .

This paper is organized as follows. In Section 2, we present some notations and preliminaries about Hardy spaces, new atoms, BMO type spaces and SchrödingerCalderón-Zygmund operators. In Section 3, we state the main results: two decomposition theorems (Theorem 3.1 and Theorem 3.4), Hardy estimates for commutators of Schrödinger-Calderón-Zygmund operators and the commutators of the Riesz transforms (Theorem 3.5 and Theorem 3.6). In Section 4, we give some examples of fundamental operators related to $L$ which are in the class $\mathcal{K}_{L}$. Section 5 is devoted to the proofs of the main theorems. Section 6 is devoted to the proofs of the key lemmas. Finally, in Section 7, we give some examples of subspaces of $H_{L}^{1}\left(\mathbb{R}^{d}\right)$ such that all commutators $[b, T], T \in \mathcal{K}_{L}$, map continuously these spaces into $L^{1}\left(\mathbb{R}^{d}\right)$.

Throughout the whole paper, $C$ denotes a positive geometric constant which is independent of the main parameters, but may change from line to line. The symbol $f \approx g$ means that $f$ is equivalent to $g$ (i.e. $C^{-1} f \leq g \leq C f$ ). In $\mathbb{R}^{d}$, we denote by $B=B(x, r)$ an open ball with center $x$ and radius $r>0$, and $t B(x, r):=B(x, t r)$ whenever $t>0$. For any measurable set $E$, we denote by $\chi_{E}$ its characteristic function, by $|E|$ its Lebesgue measure, and by $E^{c}$ the set $\mathbb{R}^{d} \backslash E$. 
Acknowledgements. The author is deeply indebted to Aline Bonami, Sandrine Grellier and Frédéric Bernicot for many helpful suggestions and discussions. He also thanks Sandrine Grellier for her carefully reading and revision of the manuscript. He would like to thank the referee for helpful suggestions and comments. Finally, he would like to thank the Vietnam Institute for Advanced Study in Mathematics for financial support and hospitality.

\section{Some preliminaries and notations}

In this paper, we consider the Schrödinger differential operator

$$
L=-\Delta+V
$$

on $\mathbb{R}^{d}, d \geq 3$, where $V$ is a nonnegative potential, $V \neq 0$. As in the works of Dziubański et al [15], [16], we always assume that $V$ belongs to the reverse Hölder class $R H_{d / 2}$. Recall that a nonnegative locally integrable function $V$ is said to belong to a reverse Hölder class $R H_{q}, 1<q<\infty$, if there exists $C>0$ such that

$$
\left(\frac{1}{|B|} \int_{B}(V(x))^{q} d x\right)^{1 / q} \leq \frac{C}{|B|} \int_{B} V(x) d x
$$

holds for every balls $B$ in $\mathbb{R}^{d}$. By Hölder inequality, $R H_{q_{1}} \subset R H_{q_{2}}$ if $q_{1} \geq q_{2}>1$. For $q>1$, it is well-known that $V \in R H_{q}$ implies $V \in R H_{q+\varepsilon}$ for some $\varepsilon>0$ (see [19]). Moreover, $V(y) d y$ is a doubling measure, namely for any ball $B(x, r)$ we have

$$
\int_{B(x, 2 r)} V(y) d y \leq C_{0} \int_{B(x, r)} V(y) d y .
$$

Let $\left\{T_{t}\right\}_{t>0}$ be the semigroup generated by $L$ and $T_{t}(x, y)$ be their kernels. Namely,

$$
T_{t} f(x)=e^{-t L} f(x)=\int_{\mathbb{R}^{d}} T_{t}(x, y) f(y) d y, \quad f \in L^{2}\left(\mathbb{R}^{d}\right), \quad t>0 .
$$

We say that a function $f \in L^{2}\left(\mathbb{R}^{d}\right)$ belongs to the space $\mathbb{H}_{L}^{1}\left(\mathbb{R}^{d}\right)$ if

$$
\|f\|_{\mathbb{H}_{L}^{1}}:=\left\|\mathcal{M}_{L} f\right\|_{L^{1}}<\infty
$$

where $\mathcal{M}_{L} f(x):=\sup _{t>0}\left|T_{t} f(x)\right|$ for all $x \in \mathbb{R}^{d}$. The space $H_{L}^{1}\left(\mathbb{R}^{d}\right)$ is then defined as the completion of $\mathbb{H}_{L}^{1}\left(\mathbb{R}^{d}\right)$ with respect to this norm.

In [15] it was shown that the dual of $H_{L}^{1}\left(\mathbb{R}^{d}\right)$ can be identified with the space $\mathrm{BMO}_{L}\left(\mathbb{R}^{d}\right)$ which consists of all functions $f \in \operatorname{BMO}\left(\mathbb{R}^{d}\right)$ with

$$
\|f\|_{\mathrm{BMO}_{L}}:=\|f\|_{\mathrm{BMO}}+\sup _{\rho(x) \leq r} \frac{1}{|B(x, r)|} \int_{B(x, r)}|f(y)| d y<\infty,
$$


where $\rho$ is the auxiliary function defined as in [39], that is,

$$
\rho(x)=\sup \left\{r>0: \frac{1}{r^{d-2}} \int_{B(x, r)} V(y) d y \leq 1\right\},
$$

$x \in \mathbb{R}^{d}$. Clearly, $0<\rho(x)<\infty$ for all $x \in \mathbb{R}^{d}$, and thus $\mathbb{R}^{d}=\bigcup_{n \in \mathbb{Z}} \mathcal{B}_{n}$, where the sets $\mathcal{B}_{n}$ are defined by

$$
\mathcal{B}_{n}=\left\{x \in \mathbb{R}^{d}: 2^{-(n+1) / 2}<\rho(x) \leq 2^{-n / 2}\right\} .
$$

The following proposition plays an important role in our study.

Proposition 2.1 (see [39], Lemma 1.4). There exist two constants $\kappa>1$ and $k_{0} \geq 1$ such that for all $x, y \in \mathbb{R}^{d}$,

$$
\kappa^{-1} \rho(x)\left(1+\frac{|x-y|}{\rho(x)}\right)^{-k_{0}} \leq \rho(y) \leq \kappa \rho(x)\left(1+\frac{|x-y|}{\rho(x)}\right)^{k_{0} /\left(k_{0}+1\right)} .
$$

Throughout the whole paper, we denote by $\mathcal{C}_{L}$ the $L$-constant

$$
\mathcal{C}_{L}=8.9^{k_{0}} \kappa
$$

where $k_{0}$ and $\kappa$ are defined as in Proposition 2.1.

Given $1<q \leq \infty$. Following Dziubański and Zienkiewicz [16], a function $a$ is called a $\left(H_{L}^{1}, q\right)$-atom related to the ball $B\left(x_{0}, r\right)$ if $r \leq \mathcal{C}_{L} \rho\left(x_{0}\right)$ and

(i) supp $a \subset B\left(x_{0}, r\right)$,

(ii) $\|a\|_{L^{q}} \leq\left|B\left(x_{0}, r\right)\right|^{1 / q-1}$,

(iii) if $r \leq \frac{1}{\mathcal{C}_{L}} \rho\left(x_{0}\right)$ then $\int_{\mathbb{R}^{d}} a(x) d x=0$.

A function $a$ is called a classical $\left(H^{1}, q\right)$-atom related to the ball $B=B\left(x_{0}, r\right)$ if it satisfies (i), (ii) and $\int_{\mathbb{R}^{d}} a(x) d x=0$.

The following atomic characterization of $H_{L}^{1}\left(\mathbb{R}^{d}\right)$ is due to [16].

Theorem 2.2 (see [16], Theorem 1.5). Let $1<q \leq \infty$. A function $f$ is in $H_{L}^{1}\left(\mathbb{R}^{d}\right)$ if and only if it can be written as $f=\sum_{j} \lambda_{j} a_{j}$, where $a_{j}$ are $\left(H_{L}^{1}, q\right)$-atoms and $\sum_{j}\left|\lambda_{j}\right|<\infty$. Moreover,

$$
\|f\|_{H_{L}^{1}} \approx \inf \left\{\sum_{j}\left|\lambda_{j}\right|: f=\sum_{j} \lambda_{j} a_{j}\right\} .
$$

Note that a classical $\left(H^{1}, q\right)$-atom is not a $\left(H_{L}^{1}, q\right)$-atom in general. In fact, there exists a constant $C>0$ such that if $f$ is a classical $\left(H^{1}, q\right)$-atom, then it can be written as $f=\sum_{j=1}^{n} \lambda_{j} a_{j}$, for some $n \in \mathbb{Z}^{+}$, where $a_{j}$ are $\left(H_{L}^{1}, q\right)$-atoms and $\sum_{j=1}^{n}\left|\lambda_{j}\right| \leq C$, see for example [47]. In this work, we need a variant of the definition of atoms for $H_{L}^{1}\left(\mathbb{R}^{d}\right)$ which include classical $\left(H^{1}, q\right)$-atoms and $\left(H_{L}^{1}, q\right)$ atoms. This kind of atoms have been used in the work of Chang, Dafni and Stein, see $[11],[13]$. 
Definition 2.3. Given $1<q \leq \infty$ and $\varepsilon>0$. A function $a$ is called a generalized $\left(H_{L}^{1}, q, \varepsilon\right)$-atom related to the ball $B\left(x_{0}, r\right)$ if

(i) $\operatorname{supp} a \subset B\left(x_{0}, r\right)$,

(ii) $\|a\|_{L^{q}} \leq\left|B\left(x_{0}, r\right)\right|^{1 / q-1}$,

(iii) $\left|\int_{\mathbb{R}^{d}} a(x) d x\right| \leq\left(\frac{r}{\rho\left(x_{0}\right)}\right)^{\varepsilon}$.

The space $\mathbb{H}_{L, a t}^{1, q, \varepsilon}\left(\mathbb{R}^{d}\right)$ is defined to be set of all functions $f$ in $L^{1}\left(\mathbb{R}^{d}\right)$ which can be written as $f=\sum_{j=1}^{\infty} \lambda_{j} a_{j}$ where the $a_{j}$ are generalized $\left(H_{L}^{1}, q, \varepsilon\right)$-atoms and the $\lambda_{j}$ are complex numbers such that $\sum_{j=1}^{\infty}\left|\lambda_{j}\right|<\infty$. As usual, the norm on $\mathbb{H}_{L, a t}^{1, q, \varepsilon}\left(\mathbb{R}^{d}\right)$ is defined by

$$
\|f\|_{\mathbb{H}_{L, a t}^{1, q, \varepsilon}}=\inf \left\{\sum_{j=1}^{\infty}\left|\lambda_{j}\right|: f=\sum_{j=1}^{\infty} \lambda_{j} a_{j}\right\}
$$

The space $\mathbb{H}_{L, \text { fin }}^{1, q, \varepsilon}\left(\mathbb{R}^{d}\right)$ is defined to be set of all $f=\sum_{j=1}^{k} \lambda_{j} a_{j}$, where the $a_{j}$ are generalized $\left(H_{L}^{1}, q, \varepsilon\right)$-atoms. Then, the norm of $f$ in $\mathbb{H}_{L, \text {,in }}^{1, q, \varepsilon}\left(\mathbb{R}^{d}\right)$ is defined by

$$
\|f\|_{\mathbb{H}_{L, \text { fin }}^{1, q, \varepsilon}}=\inf \left\{\sum_{j=1}^{k}\left|\lambda_{j}\right|: f=\sum_{j=1}^{k} \lambda_{j} a_{j}\right\}
$$

Remark 2.4. Let $1<q \leq \infty$ and $\varepsilon>0$. Then, a classical $\left(H^{1}, q\right)$-atom is a generalized $\left(H_{L}^{1}, q, \varepsilon\right)$-atom related to the same ball, and a $\left(H_{L}^{1}, q\right)$-atom is $\mathcal{C}_{L} \varepsilon$ times a generalized $\left(H_{L}^{1}, q, \varepsilon\right)$-atom related to the same ball.

Throughout the whole paper, we always use generalized $\left(H_{L}^{1}, q, \varepsilon\right)$-atoms except in the proof of Theorem 3.6. More precisely, in order to prove Theorem 3.6, we need to use $\left(H_{L}^{1}, q\right)$-atoms from Dziubański and Zienkiewicz (see above).

The following gives a characterization of $H_{L}^{1}\left(\mathbb{R}^{n}\right)$ in terms of generalized atoms.

Theorem 2.5. Let $1<q \leq \infty$ and $\varepsilon>0$. Then, $\mathbb{H}_{L, a t}^{1, q, \varepsilon}\left(\mathbb{R}^{d}\right)=H_{L}^{1}\left(\mathbb{R}^{d}\right)$ and the norms are equivalent.

In order to prove Theorem 2.5, we need the following lemma.

Lemma 2.6 (see [31], Lemma 2). Let $V \in R H_{d / 2}$. Then, there exists $\sigma_{0}>0$ depends only on $L$, such that for every $|y-z|<|x-y| / 2$ and $t>0$, we have

$$
\left|T_{t}(x, y)-T_{t}(x, z)\right| \leq C\left(\frac{|y-z|}{\sqrt{t}}\right)^{\sigma_{0}} t^{-d / 2} e^{-|x-y|^{2} / t} \leq C \frac{|y-z|^{\sigma_{0}}}{|x-y|^{d+\sigma_{0}}} .
$$

Proof of Theorem 2.5. As $\mathcal{M}_{L}$ is a sublinear operator, by Remark 2.4 and Theorem 2.2 , it is sufficient to show that

$$
\left\|\mathcal{M}_{L}(a)\right\|_{L^{1}} \leq C
$$

for all generalized $\left(H_{L}^{1}, q, \varepsilon\right)$-atom $a$ related to the ball $B=B\left(x_{0}, r\right)$. 
Indeed, from the $L^{q}$-boundedness of the classical Hardy-Littlewood maximal operator $\mathcal{M}$, the estimate $\mathcal{M}_{L}(a) \leq C \mathcal{M}(a)$ and Hölder inequality,

$$
\left\|\mathcal{M}_{L}(a)\right\|_{L^{1}(2 B)} \leq C\|\mathcal{M}(a)\|_{L^{1}(2 B)} \leq C|2 B|^{1 / q^{\prime}}\|\mathcal{M}(a)\|_{L^{q}} \leq C,
$$

where $1 / q^{\prime}+1 / q=1$. Let $x \notin 2 B$ and $t>0$, Lemma 2.6 and (3.5) of [16] give

$$
\begin{aligned}
\left|T_{t}(a)(x)\right| & =\left|\int_{\mathbb{R}^{d}} T_{t}(x, y) a(y) d y\right| \\
& \leq\left|\int_{B}\left(T_{t}(x, y)-T_{t}\left(x, x_{0}\right)\right) a(y) d y\right|+\left|T_{t}\left(x, x_{0}\right)\right|\left|\int_{B} a(y) d y\right| \\
& \leq C \frac{r^{\sigma_{0}}}{\left|x-x_{0}\right|^{d+\sigma_{0}}}+C \frac{r^{\varepsilon}}{\left|x-x_{0}\right|^{d+\varepsilon}} .
\end{aligned}
$$

Therefore,

$$
\begin{aligned}
\left\|\mathcal{M}_{L}(a)\right\|_{L^{1}\left((2 B)^{c}\right)} & =\left\|\sup _{t>0}\left|T_{t}(a)\right|\right\|_{L^{1}\left((2 B)^{c}\right)} \\
& \leq C \int_{(2 B)^{c}} \frac{r^{\sigma_{0}}}{\left|x-x_{0}\right|^{d+\sigma_{0}}} d x+C \int_{(2 B)^{c}} \frac{r^{\varepsilon}}{\left|x-x_{0}\right|^{d+\varepsilon}} d x \leq C .
\end{aligned}
$$

Then, (2.5) follows from (2.6) and (2.7).

By Theorem 2.5, the following can be seen as a direct consequence of Proposition 3.2 of [47] and Remark 2.4.

Proposition 2.7. Let $1<q<\infty, \varepsilon>0$ and $\mathcal{X}$ be a Banach space. Suppose that $T: \mathbb{H}_{L, \text { fin }}^{1, q, \varepsilon}\left(\mathbb{R}^{d}\right) \rightarrow \mathcal{X}$ is a sublinear operator with

$$
\sup \left\{\|T a\|_{\mathcal{X}}: \text { a is a generalized }\left(H_{L}^{1}, q, \varepsilon\right)-\text { atom }\right\}<\infty \text {. }
$$

Then, $T$ can be extended to a bounded sublinear operator $\widetilde{T}$ from $H_{L}^{1}\left(\mathbb{R}^{d}\right)$ into $\mathcal{X}$. Moreover,

$$
\|\widetilde{T}\|_{H_{L}^{1} \rightarrow \mathcal{X}} \leq C \sup \left\{\|T a\|_{\mathcal{X}}: \text { a is a generalized }\left(H_{L}^{1}, q, \varepsilon\right)-\text { atom }\right\} .
$$

Now, we turn to explain the new BMO type spaces introduced by Bongioanni, Harboure and Salinas in [7]. Here and in what follows $f_{B}:=\frac{1}{|B|} \int_{B} f(x) d x$ and

$$
M O(f, B):=\frac{1}{|B|} \int_{B}\left|f(y)-f_{B}\right| d y .
$$

For $\theta \geq 0$, following [7], we denote by $\mathrm{BMO}_{L, \theta}\left(\mathbb{R}^{d}\right)$ the set of all locally integrable functions $f$ such that

$$
\|f\|_{\mathrm{BMO}_{L, \theta}}=\sup _{B(x, r)}\left(\frac{1}{(1+r / \rho(x))^{\theta}} M O(f, B(x, r))\right)<\infty,
$$


and $\mathrm{BMO}_{L, \theta}^{\log }\left(\mathbb{R}^{d}\right)$ the set of all locally integrable functions $f$ such that

$$
\|f\|_{\mathrm{BMO}_{L, \theta}^{\log }}=\sup _{B(x, r)}\left(\frac{\log (e+\rho(x) / r)}{(1+r / \rho(x))^{\theta}} M O(g, B(x, r))\right)<\infty .
$$

When $\theta=0$, we write $\mathrm{BMO}_{L}^{\log }\left(\mathbb{R}^{d}\right)$ instead of $\mathrm{BMO}_{L, 0}^{\log }\left(\mathbb{R}^{d}\right)$. We next define

$$
\mathrm{BMO}_{L, \infty}\left(\mathbb{R}^{d}\right)=\bigcup_{\theta \geq 0} \mathrm{BMO}_{L, \theta}\left(\mathbb{R}^{d}\right)
$$

and

$$
\mathrm{BMO}_{L, \infty}^{\log }\left(\mathbb{R}^{d}\right)=\bigcup_{\theta \geq 0} \mathrm{BMO}_{L, \theta}^{\log }\left(\mathbb{R}^{d}\right) .
$$

Observe that $\mathrm{BMO}_{L, 0}\left(\mathbb{R}^{d}\right)$ is just the classical $\mathrm{BMO}\left(\mathbb{R}^{d}\right)$ space. Moreover, for any $0 \leq \theta \leq \theta^{\prime} \leq \infty$, we have

$$
\mathrm{BMO}_{L, \theta}\left(\mathbb{R}^{d}\right) \subset \mathrm{BMO}_{L, \theta^{\prime}}\left(\mathbb{R}^{d}\right), \quad \mathrm{BMO}_{L, \theta}^{\log }\left(\mathbb{R}^{d}\right) \subset \mathrm{BMO}_{L, \theta^{\prime}}^{\log }\left(\mathbb{R}^{d}\right)
$$

and

$$
\mathrm{BMO}_{L, \theta}^{\log }\left(\mathbb{R}^{d}\right)=\mathrm{BMO}_{L, \theta}\left(\mathbb{R}^{d}\right) \cap \mathrm{BMO}_{L, \infty}^{\log }\left(\mathbb{R}^{d}\right) .
$$

Remark 2.8. The inclusions in (2.9) are strict in general. In particular:

(i) The space $\mathrm{BMO}_{L, \infty}\left(\mathbb{R}^{d}\right)$ is in general larger than the space $\mathrm{BMO}\left(\mathbb{R}^{d}\right)$. Indeed, when $V(x) \equiv|x|^{2}$, it is easy to check that the functions $b_{j}(x)=\left|x_{j}\right|^{2}$, $j=1, \ldots, d$, belong to $\mathrm{BMO}_{L, \infty}\left(\mathbb{R}^{d}\right)$ but not to $\mathrm{BMO}\left(\mathbb{R}^{d}\right)$.

(ii) The space $\mathrm{BMO}_{L, \infty}^{\log }\left(\mathbb{R}^{d}\right)$ is in general larger than the space $\mathrm{BMO}_{L}^{\log }\left(\mathbb{R}^{d}\right)$. Indeed, when $V(x) \equiv 1$, it is easy to check that the functions $b_{j}(x)=\left|x_{j}\right|, j=$ $1, \ldots, d$, belong to $\mathrm{BMO}_{L, \infty}^{\log }\left(\mathbb{R}^{d}\right)$ but not to $\mathrm{BMO}_{L}^{\log }\left(\mathbb{R}^{d}\right)$.

Next, let us recall the notation of Schrödinger-Calderón-Zygmund operators.

Let $\delta \in(0,1]$. According to [33], a continuous function $K: \mathbb{R}^{d} \times \mathbb{R}^{d} \backslash\{(x, x)$ : $\left.x \in \mathbb{R}^{d}\right\} \rightarrow \mathbb{C}$ is said to be a $(\delta, L)$-Calderón-Zygmund singular integral kernel if for each $N>0$,

$$
|K(x, y)| \leq \frac{C(N)}{|x-y|^{d}}\left(1+\frac{|x-y|}{\rho(x)}\right)^{-N}
$$

for all $x \neq y$, and

$$
\left|K(x, y)-K\left(x^{\prime}, y\right)\right|+\left|K(y, x)-K\left(y, x^{\prime}\right)\right| \leq C \frac{\left|x-x^{\prime}\right|^{\delta}}{|x-y|^{d+\delta}}
$$

for all $2\left|x-x^{\prime}\right| \leq|x-y|$.

As usual, we denote by $C_{c}^{\infty}\left(\mathbb{R}^{d}\right)$ the space of all $C^{\infty}$-functions with compact support, by $\mathcal{S}\left(\mathbb{R}^{d}\right)$ the Schwartz space on $\mathbb{R}^{d}$. 
Definition 2.9. A linear operator $T: \mathcal{S}\left(\mathbb{R}^{d}\right) \rightarrow \mathcal{S}^{\prime}\left(\mathbb{R}^{d}\right)$ is said to be a $(\delta, L)$ Calderón-Zygmund operator if $T$ can be extended to a bounded operator on $L^{2}\left(\mathbb{R}^{d}\right)$ and if there exists a $(\delta, L)$-Calderón-Zygmund singular integral kernel $K$ such that for all $f \in C_{c}^{\infty}\left(\mathbb{R}^{d}\right)$ and all $x \notin \operatorname{supp} f$, we have

$$
T f(x)=\int_{\mathbb{R}^{d}} K(x, y) f(y) d y .
$$

An operator $T$ is said to be a Schrödinger-Calderón-Zygmund operator associated with $L$ (or $L$-Calderón-Zygmund operator) if it is a $(\delta, L)$-Calderón-Zygmund operator for some $\delta \in(0,1]$. We say that $T$ satisfies the condition $T^{*} 1=0$ if there are $q \in(1, \infty]$ and $\varepsilon>0$ so that $\int_{\mathbb{R}^{d}} T a(x) d x=0$ holds for every generalized $\left(H_{L}^{1}, q, \varepsilon\right)$-atoms $a$.

Remark 2.10. (i) Using Proposition 2.1, inequality (2.11) is equivalent to

$$
|K(x, y)| \leq \frac{C(N)}{|x-y|^{d}}\left(1+\frac{|x-y|}{\rho(y)}\right)^{-N}
$$

for all $x \neq y$.

(ii) By Theorem 0.8 of [39] and Theorem 1.1 of [40], we see that the Riesz transforms $R_{j}$ are the $L$-Calderón-Zygmund operators satisfying $R_{j}^{*} 1=0$ whenever $V \in R H_{d}$.

(iii) If $T$ is a $L$-Calderón-Zygmund operator then it is also a classical CalderónZygmund operator, and thus $T$ is bounded on $L^{p}\left(\mathbb{R}^{d}\right)$ for $1<p<\infty$ and bounded from $L^{1}\left(\mathbb{R}^{d}\right)$ into $L^{1, \infty}\left(\mathbb{R}^{d}\right)$.

\section{Statement of the results}

Recall that $\mathcal{K}_{L}$ is the set of all sublinear operators $T$ bounded from $H_{L}^{1}\left(\mathbb{R}^{d}\right)$ into $L^{1}\left(\mathbb{R}^{d}\right)$ and that there are $q \in(1, \infty]$ and $\varepsilon>0$ such that

$$
\left\|\left(b-b_{B}\right) T a\right\|_{L^{1}} \leq C\|b\|_{\mathrm{BMO}}
$$

for all $b \in \operatorname{BMO}\left(\mathbb{R}^{d}\right)$, any generalized $\left(H_{L}^{1}, q, \varepsilon\right)$-atom $a$ related to the ball $B$, where $C>0$ is a constant independent of $b, a$.

\subsection{Two decomposition theorems}

Let $b$ be a locally integrable function and $T \in \mathcal{K}_{L}$. As usual, the (sublinear) commutator $[b, T]$ of the operator $T$ is defined by $[b, T](f)(x):=T((b(x)-b(\cdot)) f(\cdot))(x)$.

Theorem 3.1 (Subbilinear decomposition). Let $T \in \mathcal{K}_{L}$. There exists a bounded subbilinear operator $\mathfrak{R}=\mathfrak{R}_{T}: H_{L}^{1}\left(\mathbb{R}^{d}\right) \times \mathrm{BMO}\left(\mathbb{R}^{d}\right) \rightarrow L^{1}\left(\mathbb{R}^{d}\right)$ such that for all $(f, b) \in H_{L}^{1}\left(\mathbb{R}^{d}\right) \times \operatorname{BMO}\left(\mathbb{R}^{d}\right)$, we have

$$
|T(\mathfrak{S}(f, b))|-\mathfrak{R}(f, b) \leq|[b, T](f)| \leq \mathfrak{R}(f, b)+|T(\mathfrak{S}(f, b))|,
$$

where $\mathfrak{S}$ is a bounded bilinear operator from $H_{L}^{1}\left(\mathbb{R}^{d}\right) \times \mathrm{BMO}\left(\mathbb{R}^{d}\right)$ into $L^{1}\left(\mathbb{R}^{d}\right)$ which does not depend on $T$. 
Using Theorem 3.1, we obtain immediately the following result.

Proposition 3.2. Let $T \in \mathcal{K}_{L}$ so that $T$ is of weak type $(1,1)$. Then, the subbilinear operator $\mathfrak{T}(f, g)=[g, T](f)$ maps continuously $H_{L}^{1}\left(\mathbb{R}^{d}\right) \times \mathrm{BMO}\left(\mathbb{R}^{d}\right)$ into $L^{1, \infty}\left(\mathbb{R}^{d}\right)$.

As the Riesz transforms $R_{j}=\partial_{x_{j}} L^{-1 / 2}$ are of weak type $(1,1)$ (see [30]), the following can be seen as a consequence of Proposition 3.2 (see also [32]).

Corollary 3.3 (see [32], Theorem 4.1). Let $b \in \mathrm{BMO}\left(\mathbb{R}^{d}\right)$. Then, the commutators $\left[b, R_{j}\right]$ are bounded from $H_{L}^{1}\left(\mathbb{R}^{d}\right)$ into $L^{1, \infty}\left(\mathbb{R}^{d}\right)$.

When $T$ is linear and belongs to $\mathcal{K}_{L}$, we obtain the bilinear decomposition for the linear commutator $[b, T]$ of $f,[b, T](f)=b T(f)-T(b f)$, instead of the subbilinear decomposition as stated in Theorem 3.1.

Theorem 3.4 (Bilinear decomposition). Let $T$ be a linear operator in $\mathcal{K}_{L}$. Then, there exists a bounded bilinear operator $\mathfrak{R}=\mathfrak{R}_{T}: H_{L}^{1}\left(\mathbb{R}^{d}\right) \times \mathrm{BMO}\left(\mathbb{R}^{d}\right) \rightarrow L^{1}\left(\mathbb{R}^{d}\right)$ such that for all $(f, b) \in H_{L}^{1}\left(\mathbb{R}^{d}\right) \times \mathrm{BMO}\left(\mathbb{R}^{d}\right)$, we have

$$
[b, T](f)=\mathfrak{R}(f, b)+T(\mathfrak{S}(f, b)),
$$

where $\mathfrak{S}$ is as in Theorem 3.1 .

\subsection{Hardy estimates for linear commutators}

Our first main result of this subsection is the following theorem.

Theorem 3.5. (i) Let $b \in \mathrm{BMO}_{L}^{\log }\left(\mathbb{R}^{d}\right)$ and $T$ be a L-Calderón-Zygmund operator satisfying $T^{*} 1=0$. Then, the linear commutator $[b, T]$ is bounded on $H_{L}^{1}\left(\mathbb{R}^{d}\right)$.

(ii) When $V \in R H_{d}$, the converse holds. Namely, if $b \in \operatorname{BMO}\left(\mathbb{R}^{d}\right)$ and $[b, T]$ is bounded on $H_{L}^{1}\left(\mathbb{R}^{d}\right)$ for every $L$-Calderón-Zygmund operator $T$ satisfying $T^{*} 1=0$, then $b \in \mathrm{BMO}_{L}^{\log }\left(\mathbb{R}^{d}\right)$. Furthermore,

$$
\|b\|_{\mathrm{BMO}_{L}^{\log }} \approx\|b\|_{\mathrm{BMO}}+\sum_{j=1}^{d}\left\|\left[b, R_{j}\right]\right\|_{H_{L}^{1} \rightarrow H_{L}^{1}} .
$$

Next result concerns the $H_{L}^{1}$-estimates for commutators of the Riesz transforms.

Theorem 3.6. Let $b \in \mathrm{BMO}_{L, \infty}\left(\mathbb{R}^{d}\right)$. Then, the commutators $\left[b, R_{j}\right], j=$ $1, \ldots, d$, are bounded on $H_{L}^{1}\left(\mathbb{R}^{d}\right)$ if and only if $b \in \mathrm{BMO}_{L, \infty}^{\log }\left(\mathbb{R}^{d}\right)$. Furthermore, if $b \in \mathrm{BMO}_{L, \theta}^{\log }\left(\mathbb{R}^{d}\right)$ for some $\theta \geq 0$, we have

$$
\|b\|_{\mathrm{BMO}_{L, \theta}^{\log }} \approx\|b\|_{\mathrm{BMO}_{L, \theta}}+\sum_{j=1}^{d}\left\|\left[b, R_{j}\right]\right\|_{H_{L}^{1} \rightarrow H_{L}^{1}} .
$$

Remark that the above constants depend on $\theta$. 
Note that $\mathrm{BMO}_{L}^{\log }\left(\mathbb{R}^{d}\right)$ is in general proper subset of $\mathrm{BMO}_{L, \infty}^{\log }\left(\mathbb{R}^{d}\right)$ (see Remark 2.8). When $V \in R H_{d}$, although the Riesz transforms $R_{j}$ are $L$-CalderónZygmund operators satisfying $R_{j}^{*} 1=0$, Theorem 3.6 cannot be deduced from Theorem 3.5.

As a consequence of Theorem 3.6, we obtain the following interesting result.

Corollary 3.7. Let $b \in \mathrm{BMO}\left(\mathbb{R}^{d}\right)$. Then, $b$ belongs to $\operatorname{LMO}\left(\mathbb{R}^{d}\right)$ if and only if the vector-valued commutator $\left[b, \nabla(-\Delta+1)^{-1 / 2}\right]$ maps continuously $h^{1}\left(\mathbb{R}^{d}\right)$ into $h^{1}\left(\mathbb{R}^{d}, \mathbb{R}^{d}\right)=\left(h^{1}\left(\mathbb{R}^{d}\right), \ldots, h^{1}\left(\mathbb{R}^{d}\right)\right)$. Furthermore,

$$
\|b\|_{\mathrm{LMO}} \approx\|b\|_{\mathrm{BMO}}+\left\|\left[b, \nabla(-\Delta+1)^{-1 / 2}\right]\right\|_{h^{1}\left(\mathbb{R}^{d}\right) \rightarrow h^{1}\left(\mathbb{R}^{d}, \mathbb{R}^{d}\right)} .
$$

Here $h^{1}\left(\mathbb{R}^{d}\right)$ is the local Hardy space of D. Goldberg (see $\left.[20]\right)$, and $\operatorname{LMO}\left(\mathbb{R}^{d}\right)$ is the space of all locally integrable functions $f$ such that

$$
\|f\|_{\mathrm{LMO}}:=\sup _{B(x, r)}\left(\log \left(e+\frac{1}{r}\right) \mathrm{MO}(f, B(x, r))\right)<\infty .
$$

It should be pointed out that LMO type spaces appear naturally when studying the boundedness of Hankel operators on the Hardy spaces $H^{1}\left(\mathbb{T}^{d}\right)$ and $H^{1}\left(\mathbb{B}^{d}\right)$ (where $\mathbb{B}^{d}$ is the unit ball in $\mathbb{C}^{d}$ and $\mathbb{T}^{d}=\partial \mathbb{B}^{d}$ ), characterizations of pointwise multipliers for BMO type spaces, endpoint estimates for commutators of singular integrals operators and their applications to PDEs, see for example [5], [9], [24], [25], [28], [36], [41], and [42].

\section{Some fundamental operators and the class $\mathcal{K}_{L}$}

The purpose of this section is to give some examples of fundamental operators related to $L$ which are in the class $\mathcal{K}_{L}$.

\subsection{The Schrödinger-Calderón-Zygmund operators}

Proposition 4.1. Let $T$ be any $L$-Calderón-Zygmund operator. Then, $T$ belongs to the class $\mathcal{K}_{L}$.

Proposition 4.2. The Riesz transforms $R_{j}$ are in the class $\mathcal{K}_{L}$.

The proof of Proposition 4.2 follows directly from Lemma 5.13 and the fact that the Riesz transforms $R_{j}$ are bounded from $H_{L}^{1}\left(\mathbb{R}^{d}\right)$ into $L^{1}\left(\mathbb{R}^{d}\right)$.

To prove Proposition 4.1, we need the following two lemmas.

Lemma 4.3. Let $1 \leq q<\infty$. Then, there exists a constant $C>0$ such that for every ball $B, f \in \operatorname{BMO}\left(\mathbb{R}^{d}\right)$ and $k \in \mathbb{Z}^{+}$,

$$
\left(\frac{1}{\left|2^{k} B\right|} \int_{2^{k} B}\left|f(y)-f_{B}\right|^{q} d y\right)^{1 / q} \leq C k\|f\|_{\mathrm{BMO}} .
$$


The proof of Lemma 4.3 follows directly from the classical John-Nirenberg inequality. See also Lemma 6.6 below.

Lemma 4.4. Let $1<q \leq \infty$ and $\varepsilon>0$. Assume that $T$ is a $(\delta, L)$-CalderónZygmund operator and $a$ is a generalized $\left(H_{L}^{1}, q, \varepsilon\right)$-atom related to the ball $B=$ $B\left(x_{0}, r\right)$. Then,

$$
\|T a\|_{L^{q}\left(2^{k+1} B \backslash 2^{k} B\right)} \leq C 2^{-k \delta_{0}}\left|2^{k} B\right|^{1 / q-1}
$$

for all $k=1,2, \ldots$, where $\delta_{0}=\min \{\varepsilon, \delta\}$.

Proof. Let $x \in 2^{k+1} B \backslash 2^{k} B$, so that $\left|x-x_{0}\right| \geq 2 r$. Since $T$ is a $(\delta, L)$-CalderónZygmund operator, we get

$$
\begin{aligned}
|T a(x)| & \leq\left|\int_{B}\left(K(x, y)-K\left(x, x_{0}\right)\right) a(y) d y\right|+\left|K\left(x, x_{0}\right)\right|\left|\int_{\mathbb{R}^{d}} a(y) d y\right| \\
& \leq C \int_{B} \frac{\left|y-x_{0}\right|^{\delta}}{\left|x-x_{0}\right|^{d+\delta}}|a(y)| d y+C \frac{1}{\left|x-x_{0}\right|^{d}}\left(1+\frac{\left|x-x_{0}\right|}{\rho\left(x_{0}\right)}\right)^{-\varepsilon}\left(\frac{r}{\rho\left(x_{0}\right)}\right)^{\varepsilon} \\
& \leq C \frac{r^{\delta}}{\left|x-x_{0}\right|^{d+\delta}}+C \frac{r^{\varepsilon}}{\left|x-x_{0}\right|^{d+\varepsilon}} \leq C \frac{r^{\delta_{0}}}{\left|x-x_{0}\right|^{d+\delta_{0}}} .
\end{aligned}
$$

Consequently,

$$
\|T a\|_{L^{q}\left(2^{k+1} B \backslash 2^{k} B\right)} \leq C \frac{r^{\delta_{0}}}{\left(2^{k} r\right)^{d+\delta_{0}}}\left|2^{k+1} B\right|^{1 / q} \leq C 2^{-k \delta_{0}}\left|2^{k} B\right|^{1 / q-1} .
$$

Proof of Proposition 4.1. Assume that $T$ is a $(\delta, L)$-Calderón-Zygmund for some $\delta \in(0,1]$. Let us first verify that $T$ is bounded from $H_{L}^{1}\left(\mathbb{R}^{d}\right)$ into $L^{1}\left(\mathbb{R}^{d}\right)$. By Proposition 2.7, it is sufficient to show that

$$
\|T a\|_{L^{1}} \leq C
$$

for all generalized $\left(H_{L}^{1}, 2, \delta\right)$-atom $a$ related to the ball $B$. Indeed, from the $L^{2}$ boundedness of $T$ and Lemma 4.4, we obtain that

$$
\begin{aligned}
\|T a\|_{L^{1}} & =\|T a\|_{L^{1}(2 B)}+\sum_{k=1}^{\infty}\|T a\|_{L^{1}\left(2^{k+1} B \backslash 2^{k} B\right)} \\
& \leq C|2 B|^{1 / 2}\|T\|_{L^{2} \rightarrow L^{2}}\|a\|_{L^{2}}+C \sum_{k=1}^{\infty}\left|2^{k+1} B\right|^{1 / 2} 2^{-k \delta}\left|2^{k} B\right|^{-1 / 2} \leq C .
\end{aligned}
$$

Let us next establish that

$$
\left\|\left(f-f_{B}\right) T a\right\|_{L^{1}} \leq C\|f\|_{\mathrm{BMO}}
$$

for all $f \in \operatorname{BMO}\left(\mathbb{R}^{d}\right)$, and for any generalized $\left(H_{L}^{1}, 2, \delta\right)$-atom $a$ related to the ball 
$B=B\left(x_{0}, r\right)$. Indeed, by Hölder's inequality, Lemma 4.3 and Lemma 4.4, we get

$$
\begin{aligned}
& \left\|\left(f-f_{B}\right) T a\right\|_{L^{1}} \\
& =\left\|\left(f-f_{B}\right) T a\right\|_{L^{1}(2 B)}+\sum_{k \geq 1}\left\|\left(f-f_{B}\right) T a\right\|_{L^{1}\left(2^{k+1} B \backslash 2^{k} B\right)} \\
& \leq\left\|\left(f-f_{B}\right) \chi_{2 B}\right\|_{L^{2}}\|T\|_{L^{2} \rightarrow L^{2}}\|a\|_{L^{2}}+\sum_{k \geq 1}\left\|f-f_{B}\right\|_{L^{2}\left(2^{k+1} B\right)}\|T a\|_{L^{2}\left(2^{k+1} B \backslash 2^{k} B\right)} \\
& \leq C\|f\|_{\mathrm{BMO}}+\sum_{k \geq 1} C(k+1)\|f\|_{\mathrm{BMO}}\left|2^{k+1} B\right|^{1 / 2} 2^{-k \delta}\left|2^{k} B\right|^{-1 / 2} \leq C\|f\|_{\mathrm{BMO}},
\end{aligned}
$$

which ends the proof.

\subsection{The $L$-maximal operators}

Recall that $\left\{T_{t}\right\}_{t>0}$ is heat semigroup generated by $L$ and $T_{t}(x, y)$ are their kernels. Namely,

$$
T_{t} f(x)=e^{-t L} f(x)=\int_{\mathbb{R}^{d}} T_{t}(x, y) f(y) d y, \quad f \in L^{2}\left(\mathbb{R}^{d}\right), \quad t>0 .
$$

Then the "heat" maximal operator is defined by

$$
\mathcal{M}_{L} f(x)=\sup _{t>0}\left|T_{t} f(x)\right|,
$$

and the "Poisson" maximal operator is defined by

$$
\mathcal{M}_{L}^{P} f(x)=\sup _{t>0}\left|P_{t} f(x)\right|,
$$

where

$$
P_{t} f(x)=e^{-t \sqrt{L}} f(x)=\frac{t}{2 \sqrt{\pi}} \int_{0}^{\infty} \frac{e^{-t^{2} /(4 u)}}{u^{3 / 2}} T_{u} f(x) d u .
$$

Proposition 4.5. The "heat" maximal operator $\mathcal{M}_{L}$ is in the class $\mathcal{K}_{L}$.

Proposition 4.6. The "Poisson" maximal operator $\mathcal{M}_{L}^{P}$ is in the class $\mathcal{K}_{L}$.

Here we just give the proof of Proposition 4.5. For the one of Proposition 4.6, we leave the details to the interested reader.

Proof of Proposition 4.5. Obviously, $\mathcal{M}_{L}$ is bounded from $H_{L}^{1}\left(\mathbb{R}^{d}\right)$ into $L^{1}\left(\mathbb{R}^{d}\right)$.

Now, let us prove that

$$
\left\|\left(f-f_{B}\right) \mathcal{M}_{L}(a)\right\|_{L^{1}} \leq C\|f\|_{\mathrm{BMO}}
$$

for all $f \in \operatorname{BMO}\left(\mathbb{R}^{d}\right)$, any generalized $\left(H_{L}^{1}, 2, \sigma_{0}\right)$-atom $a$ related to the ball $B=$ $B\left(x_{0}, r\right)$, where the constant $\sigma_{0}>0$ is as in Lemma 2.6. Indeed, by the proof of Theorem 2.5 , for every $x \notin 2 B$,

$$
\mathcal{M}_{L}(a)(x) \leq C \frac{r^{\sigma_{0}}}{\left|x-x_{0}\right|^{d+\sigma_{0}}} .
$$


Therefore, using Lemma 4.3, the $L^{2}$-boundedness of the classical Hardy-Littlewood maximal operator $\mathcal{M}$ and the estimate $\mathcal{M}_{L}(a) \leq C \mathcal{M}(a)$, we obtain that

$$
\begin{aligned}
& \left\|\left(f-f_{B}\right) \mathcal{M}_{L}(a)\right\|_{L^{1}} \\
& \quad=\left\|\left(f-f_{B}\right) \mathcal{M}_{L}(a)\right\|_{L^{1}(2 B)}+\left\|\left(f-f_{B}\right) \mathcal{M}_{L}(a)\right\|_{L^{1}\left((2 B)^{c}\right)} \\
& \quad \leq C\left\|f-f_{B}\right\|_{L^{2}(2 B)}\|\mathcal{M}(a)\|_{L^{2}}+C \int_{\left|x-x_{0}\right| \geq 2 r}\left|f(x)-f_{B\left(x_{0}, r\right)}\right| \frac{r^{\sigma_{0}}}{\left|x-x_{0}\right|^{d+\sigma_{0}}} d x \\
& \quad \leq C\|f\|_{\mathrm{BMO}},
\end{aligned}
$$

where we have used the following classical inequality:

$$
\int_{\left|x-x_{0}\right| \geq 2 r}\left|f(x)-f_{B\left(x_{0}, r\right)}\right| \frac{r^{\sigma_{0}}}{\left|x-x_{0}\right|^{d+\sigma_{0}}} d x \leq C\|f\|_{\mathrm{BMO}},
$$

which proof can be found in [17]. This completes the proof of Proposition 4.5.

\subsection{The $L$-square functions}

Recall (see [15]) that the $L$-square funcfions $\mathfrak{g}$ and $\mathcal{G}$ are defined by

$$
\mathfrak{g}(f)(x)=\left(\int_{0}^{\infty}\left|t \partial_{t} T_{t}(f)(x)\right|^{2} \frac{d t}{t}\right)^{1 / 2}
$$

and

$$
\mathcal{G}(f)(x)=\left(\int_{0}^{\infty} \int_{|x-y|<t}\left|t \partial_{t} T_{t}(f)(y)\right|^{2} \frac{d y d t}{t^{d+1}}\right)^{1 / 2} .
$$

Proposition 4.7. The L-square function $\mathfrak{g}$ is in the class $\mathcal{K}_{L}$.

Proposition 4.8. The L-square function $\mathcal{G}$ is in the class $\mathcal{K}_{L}$.

Here we just give the proof for Proposition 4.7. For the one of Proposition 4.8, we leave the details to the interested reader.

In order to prove Proposition 4.7, we need the following lemma.

Lemma 4.9. There exists a constant $C>0$ such that

$$
\left|t \partial_{t} T_{t}(x, y+h)-t \partial_{t} T_{t}(x, y)\right| \leq C\left(\frac{|h|}{\sqrt{t}}\right)^{\delta} t^{-d / 2} e^{-\frac{c}{4}|x-y|^{2} / t},
$$

for all $|h|<|x-y| / 2,0<t$. Here and in the proof of Proposition 4.7 , the constants $\delta, c \in(0,1)$ are as in Proposition 4 of [15].

Proof. One only needs to consider the case $\sqrt{t}<|h|<|x-y| / 2$. Otherwise, (4.1) follows directly from (b) in Proposition 4 of [15].

For $\sqrt{t}<|h|<|x-y| / 2$. By (a) in Proposition 4 of [15], we get

$$
\begin{aligned}
\left|t \partial_{t} T_{t}(x, y+h)-t \partial_{t} T_{t}(x, y)\right| & \leq C t^{-d / 2} e^{-c|x-y-h|^{2} / t}+C t^{-d / 2} e^{-c|x-y|^{2} / t} \\
& \leq C\left(\frac{|h|}{\sqrt{t}}\right)^{\delta} t^{-d / 2} e^{-\frac{c}{4}|x-y|^{2} / t} .
\end{aligned}
$$


Proof of Proposition 4.7. The $\left(H_{L}^{1}-L^{1}\right)$ type boundedness of $\mathfrak{g}$ is well-known, see for example [15], [22]. Let us now show that

$$
\left\|\left(f-f_{B}\right) \mathfrak{g}(a)\right\|_{L^{1}} \leq C\|f\|_{\mathrm{BMO}}
$$

for all $f \in \operatorname{BMO}\left(\mathbb{R}^{d}\right)$, any generalized $\left(H_{L}^{1}, 2, \delta\right)$-atom $a$ related to the ball $B=$ $B\left(x_{0}, r\right)$. Indeed, it follows from Lemma 4.9 and $(a)$ in Proposition 4 of [15] that for every $t>0, x \notin 2 B$,

$$
\begin{aligned}
\left|t \partial_{t} T_{t}(a)(x)\right|= & \left|\int_{B}\left(t \partial_{t} T_{t}(x, y)-t \partial_{t} T_{t}\left(x, x_{0}\right)\right) a(y) d y+t \partial_{t} T_{t}\left(x, x_{0}\right) \int_{B} a(y) d y\right| \\
\leq & C\left(\frac{r}{\sqrt{t}}\right)^{\delta} t^{-d / 2} e^{-\frac{c}{4}\left|x-x_{0}\right|^{2} / t}\|a\|_{L^{1}} \\
& +C t^{-d / 2} e^{-c\left|x-x_{0}\right|^{2} / t}\left(1+\frac{\sqrt{t}}{\rho(x)}+\frac{\sqrt{t}}{\rho\left(x_{0}\right)}\right)^{-\delta}\left(\frac{r}{\rho\left(x_{0}\right)}\right)^{\delta} \\
\leq & C\left(\frac{r}{\sqrt{t}}\right)^{\delta} t^{-d / 2} e^{-\frac{c}{4}\left|x-x_{0}\right|^{2} / t} .
\end{aligned}
$$

Therefore, as $0<\delta<1$, using the estimate $e^{-\frac{c}{2}\left|x-x_{0}\right|^{2} / t} \leq C(c, d)\left(\frac{t}{\left|x-x_{0}\right|^{2}}\right)^{d+2}$,

$$
\begin{aligned}
\mathfrak{g}(a)(x) & \leq C\left\{\int_{0}^{\infty}\left(\frac{r^{2}}{t}\right)^{\delta} t^{-d} e^{-\frac{c}{2}\left|x-x_{0}\right|^{2} / t} \frac{d t}{t}\right\}^{1 / 2} \\
& \leq C\left\{\int_{0}^{\left|x-x_{0}\right|^{2}}\left(\frac{r^{2}}{t}\right)^{\delta} t^{-d}\left(\frac{t}{\left|x-x_{0}\right|^{2}}\right)^{d+2} \frac{d t}{t}+\int_{\left|x-x_{0}\right|^{2}}^{\infty}\left(\frac{r^{2}}{t}\right)^{\delta} t^{-d} \frac{d t}{t}\right\}^{1 / 2} \\
& \leq C \frac{r^{\delta}}{\left|x-x_{0}\right|^{d+\delta}} .
\end{aligned}
$$

Therefore, the $L^{2}$-boundedness of $\mathfrak{g}$ and Lemma 4.3 yield

$$
\begin{aligned}
\|(f- & \left.f_{B}\right) \mathfrak{g}(a)\left\|_{L^{1}}=\right\|\left(f-f_{B}\right) \mathfrak{g}(a)\left\|_{L^{1}(2 B)}+\right\|\left(f-f_{B}\right) \mathfrak{g}(a) \|_{L^{1}\left((2 B)^{c}\right)} \\
& \leq\left\|f-f_{B}\right\|_{L^{2}(2 B)}\|\mathfrak{g}(a)\|_{L^{2}}+C \int_{\left|x-x_{0}\right| \geq 2 r}\left|f(x)-f_{B\left(x_{0}, r\right)}\right| \frac{r^{\delta}}{\left|x-x_{0}\right|^{d+\delta}} d x \\
& \leq C\|f\|_{\mathrm{BMO}},
\end{aligned}
$$

which ends the proof.

\section{Proof of the main results}

In this section, we fix a non-negative function $\varphi \in \mathcal{S}\left(\mathbb{R}^{d}\right)$ with $\operatorname{supp} \varphi \subset B(0,1)$ and $\int_{\mathbb{R}^{d}} \varphi(x) d x=1$. Then, we define the linear operator $\mathfrak{H}$ by

$$
\mathfrak{H}(f)=\sum_{n, k}\left(\psi_{n, k} f-\varphi_{2^{-n / 2}} *\left(\psi_{n, k} f\right)\right),
$$

where $\psi_{n, k}, n \in \mathbb{Z}, k=1,2, \ldots$ is as in Lemma 2.5 of [16] (see also Lemma 6.2).

Remark 5.1. When $V(x) \equiv 1$, we can define $\mathfrak{H}(f)=f-\varphi * f$. 
Let us now consider the set $\mathcal{E}=\{0,1\}^{d} \backslash\{(0, \cdots, 0)\}$ and $\left\{\psi^{\sigma}\right\}_{\sigma \in \mathcal{E}}$ the wavelet with compact support as in Section 3 of [4] (see also Section 2 of [28]). Suppose that $\psi^{\sigma}$ is supported in the cube $(1 / 2-c / 2,1 / 2+c / 2)^{d}$ for all $\sigma \in \mathcal{E}$. As it is classical, for $\sigma \in \mathcal{E}$ and $I$ a dyadic cube of $\mathbb{R}^{d}$ which may be written as the set of $x$ such that $2^{j} x-k \in(0,1)^{d}$, we note

$$
\psi_{I}^{\sigma}(x)=2^{d j / 2} \psi^{\sigma}\left(2^{j} x-k\right) .
$$

In the sequel, the letter $I$ always refers to dyadic cubes. Moreover, we note $k I$ the cube of same center dilated by the coefficient $k$.

Remark 5.2. For every $\sigma \in \mathcal{E}$ and $I$ a dyadic cube. Because of the assumption on the support of $\psi^{\sigma}$, the function $\psi_{I}^{\sigma}$ is supported in the cube $c I$.

In [4] (see also [28]), Bonami et al. established the following.

Proposition 5.3. The bounded bilinear operator $\Pi$, defined by

$$
\Pi(f, g)=\sum_{I} \sum_{\sigma \in \mathcal{E}}\left\langle f, \psi_{I}^{\sigma}\right\rangle\left\langle g, \psi_{I}^{\sigma}\right\rangle\left(\psi_{I}^{\sigma}\right)^{2},
$$

is bounded from $H^{1}\left(\mathbb{R}^{d}\right) \times \operatorname{BMO}\left(\mathbb{R}^{d}\right)$ into $L^{1}\left(\mathbb{R}^{d}\right)$.

\subsection{Proof of Theorem 3.1 and Theorem 3.4}

In order to prove Theorem 3.1 and Theorem 3.4, we need the following key two lemmas, whose proofs will given in Section 6.

Lemma 5.4. The linear operator $\mathfrak{H}$ is bounded from $H_{L}^{1}\left(\mathbb{R}^{d}\right)$ into $H^{1}\left(\mathbb{R}^{d}\right)$.

Lemma 5.5. Let $T \in \mathcal{K}_{L}$. Then, the subbilinear operator

$$
\mathcal{U}(f, b):=[b, T](f-\mathfrak{H}(f))
$$

is bounded from $H_{L}^{1}\left(\mathbb{R}^{d}\right) \times \operatorname{BMO}\left(\mathbb{R}^{d}\right)$ into $L^{1}\left(\mathbb{R}^{d}\right)$.

By Proposition 5.3 and Lemma 5.4, we obtain:

Proposition 5.6. The bilinear operator $\mathfrak{S}(f, g):=-\Pi(\mathfrak{H}(f), g)$ is bounded from $H_{L}^{1}\left(\mathbb{R}^{d}\right) \times \operatorname{BMO}\left(\mathbb{R}^{d}\right)$ into $L^{1}\left(\mathbb{R}^{d}\right)$.

We recall (see [28]) that the class $\mathcal{K}$ is the set of all sublinear operators $T$ bounded from $H^{1}\left(\mathbb{R}^{d}\right)$ into $L^{1}\left(\mathbb{R}^{d}\right)$ so that for some $q \in(1, \infty]$,

$$
\left\|\left(b-b_{B}\right) T a\right\|_{L^{1}} \leq C\|b\|_{\mathrm{BMO}}
$$

for all $b \in \operatorname{BMO}\left(\mathbb{R}^{d}\right)$, any classical $\left(H^{1}, q\right)$-atom $a$ related to the ball $B$, where $C>0$ a constant independent of $b, a$.

Remark 5.7. By Remark 2.4 and as $H^{1}\left(\mathbb{R}^{d}\right) \subset H_{L}^{1}\left(\mathbb{R}^{d}\right)$, we obtain that $\mathcal{K}_{L} \subset \mathcal{K}$, which allows to apply the two classical decomposition theorems (Theorem 3.1 and Theorem 3.2 of [28]). This is a key point in our proofs. 
Proof of Theorem 3.1. As $T \in \mathcal{K}_{L} \subset \mathcal{K}$, it follows from Theorem 3.1 of [28] that there exists a bounded subbilinear operator $\mathcal{V}: H^{1}\left(\mathbb{R}^{d}\right) \times \mathrm{BMO}\left(\mathbb{R}^{d}\right) \rightarrow L^{1}\left(\mathbb{R}^{d}\right)$ such that for all $(g, b) \in H^{1}\left(\mathbb{R}^{d}\right) \times \operatorname{BMO}\left(\mathbb{R}^{d}\right)$, we have

$$
|T(-\Pi(g, b))|-\mathcal{V}(g, b) \leq|[b, T](g)| \leq \mathcal{V}(g, b)+|T(-\Pi(g, b))| .
$$

Let us now define the bilinear operator $\mathfrak{R}$ by

$$
\mathfrak{R}(f, b):=|\mathcal{U}(f, b)|+\mathcal{V}(\mathfrak{H}(f), b)
$$

for all $(f, b) \in H_{L}^{1}\left(\mathbb{R}^{d}\right) \times \mathrm{BMO}\left(\mathbb{R}^{d}\right)$, where $\mathcal{U}$ is the subbilinear operator as in Lemma 5.5. Then, using the subbilinear decomposition (5.1) with $g=\mathfrak{H}(f)$,

$$
|T(\mathfrak{S}(f, b))|-\mathfrak{R}(f, b) \leq|[b, T](f)| \leq|T(\mathfrak{S}(f, b))|+\mathfrak{R}(f, b),
$$

where the bounded bilinear operator $\mathfrak{S}: H_{L}^{1}\left(\mathbb{R}^{d}\right) \times \mathrm{BMO}\left(\mathbb{R}^{d}\right) \rightarrow L^{1}\left(\mathbb{R}^{d}\right)$ is given in Proposition 5.6.

Furthermore, by Lemma 5.5 and Lemma 5.4, we get

$$
\begin{aligned}
\|\Re(f, b)\|_{L^{1}} & \leq\|\mathcal{U}(f, b)\|_{L^{1}}+\|\mathcal{V}(\mathfrak{H}(f), b)\|_{L^{1}} \\
& \leq C\|f\|_{H_{L}^{1}}\|b\|_{\mathrm{BMO}}+C\|\mathfrak{H}(f)\|_{H^{1}}\|b\|_{\mathrm{BMO}} \leq C\|f\|_{H_{L}^{1}}\|b\|_{\mathrm{BMO}},
\end{aligned}
$$

where we used the boundedness of $\mathcal{V}$ on $H^{1}\left(\mathbb{R}^{d}\right) \times \mathrm{BMO}\left(\mathbb{R}^{d}\right)$ into $L^{1}\left(\mathbb{R}^{d}\right)$. This completes the proof.

Proof of Theorem 3.4. The proof follows the same lines except that now, one deals with equalities instead of inequalities. Namely, as $T$ is a linear operator in $\mathcal{K}_{L} \subset \mathcal{K}$, Theorem 3.2 of [28] yields that there exists a bounded bilinear operator $\mathcal{W}$ : $H^{1}\left(\mathbb{R}^{d}\right) \times \mathrm{BMO}\left(\mathbb{R}^{d}\right) \rightarrow L^{1}\left(\mathbb{R}^{d}\right)$ such that for every $(g, b) \in H^{1}\left(\mathbb{R}^{d}\right) \times \mathrm{BMO}\left(\mathbb{R}^{d}\right)$,

$$
[b, T](g)=\mathcal{W}(g, b)+T(-\Pi(g, b))
$$

Therefore, for every $(f, b) \in H_{L}^{1}\left(\mathbb{R}^{d}\right) \times \operatorname{BMO}\left(\mathbb{R}^{d}\right)$,

$$
[b, T](f)=\mathfrak{R}(f, b)+T(\mathfrak{S}(f, b)),
$$

where $\mathfrak{R}(f, b):=\mathcal{U}(f, b)+\mathcal{W}(\mathfrak{H}(f), b)$ is a bounded bilinear operator from $H_{L}^{1}\left(\mathbb{R}^{d}\right) \times$ $\mathrm{BMO}\left(\mathbb{R}^{d}\right)$ into $L^{1}\left(\mathbb{R}^{d}\right)$. This completes the proof.

\subsection{Proof of Theorem 3.5 and Theorem 3.6}

First, recall that $\mathrm{VMO}_{L}\left(\mathbb{R}^{d}\right)$ is the closure of $C_{c}^{\infty}\left(\mathbb{R}^{d}\right)$ in $\mathrm{BMO}_{L}\left(\mathbb{R}^{d}\right)$. Then, the following result due to Ky [29].

Theorem 5.8. The space $H_{L}^{1}\left(\mathbb{R}^{d}\right)$ is the dual of the space $\mathrm{VMO}_{L}\left(\mathbb{R}^{d}\right)$.

In order to prove Theorem 3.5, we need the following key lemmas, whose proofs will be given in Section 6 . 
Lemma 5.9. Let $1 \leq q<\infty$ and $\theta \geq 0$. Then, for every $f \in \mathrm{BMO}_{L, \theta}^{\log }\left(\mathbb{R}^{d}\right)$, $B=B(x, r)$ and $k \in \mathbb{Z}^{+}$, we have

$$
\left(\frac{1}{\left|2^{k} B\right|} \int_{2^{k} B}\left|f(y)-f_{B}\right|^{q} d y\right)^{1 / q} \leq C k \frac{\left(1+\frac{2^{k} r}{\rho(x)}\right)^{\left(k_{0}+1\right) \theta}}{\log \left(e+\left(\frac{\rho(x)}{2^{k} r}\right)^{k_{0}+1}\right)}\|f\|_{\mathrm{BMO}_{L, \theta}^{\log },}
$$

where the constant $k_{0}$ is as in Proposition 2.1.

Lemma 5.10. Let $1<q<\infty, \varepsilon>0$ and $T$ be a L-Calderón-Zygmund operator. Then, the following two statements hold:

(i) If $T^{*} 1=0$, then $T$ is bounded from $H_{L}^{1}\left(\mathbb{R}^{d}\right)$ into $H^{1}\left(\mathbb{R}^{d}\right)$.

(ii) For every $f, g \in \mathrm{BMO}\left(\mathbb{R}^{d}\right)$, and for every generalized $\left(H_{L}^{1}, q, \varepsilon\right)$-atom a related to the ball $B$,

$$
\left\|\left(f-f_{B}\right)\left(g-g_{B}\right) T a\right\|_{L^{1}} \leq C\|f\|_{\mathrm{BMO}}\|g\|_{\mathrm{BMO}} .
$$

Proof of Theorem 3.5. (i). Assume that $T$ is a $(\delta, L)$-Calderón-Zygmund operator. We claim that, by Lemma 5.10 , it is sufficient to prove that

$$
\left\|\left(b-b_{B}\right) a\right\|_{H_{L}^{1}} \leq C\|b\|_{\mathrm{BMO}_{L}^{\log }}
$$

and

$$
\left\|\left(b-b_{B}\right) T a\right\|_{H_{L}^{1}} \leq C\|b\|_{\mathrm{BMO}_{L}^{\log }}
$$

hold for every generalized $\left(H_{L}^{1}, 2, \delta\right)$-atom $a$ related to the ball $B=B\left(x_{0}, r\right)$ with the constants are independent of $b, a$. Indeed, if (5.2) and (5.3) are true, then

$$
\begin{aligned}
& \|[b, T](a)\|_{H_{L}^{1}} \leq\left\|\left(b-b_{B}\right) T a\right\|_{H_{L}^{1}}+C\left\|T\left(\left(b-b_{B}\right) a\right)\right\|_{H^{1}} \\
& \leq C\|b\|_{\mathrm{BMO}_{L}^{\log }}+C\|T\|_{H_{L}^{1} \rightarrow H^{1}}\left\|\left(b-b_{B}\right) a\right\|_{H_{L}^{1}} \leq C\|b\|_{\mathrm{BMO}_{L}^{\log } .}
\end{aligned}
$$

Therefore, Proposition 2.7 yields that $[b, T]$ is bounded on $H_{L}^{1}\left(\mathbb{R}^{d}\right)$; moreover,

$$
\|[b, T]\|_{H_{L}^{1} \rightarrow H_{L}^{1}} \leq C
$$

where the constant $C$ is independent of $b$.

The proof of (5.2) is similar to the one of (5.3) but uses an easier argument; we leave the details to the interested reader. Let us now establish (5.3). By Theorem 5.8, it is sufficient to show that

$$
\left\|\phi\left(b-b_{B}\right) T a\right\|_{L^{1}} \leq C\|b\|_{\mathrm{BMO}_{L}^{\log }\|\phi\|_{\mathrm{BMO}_{L}}}
$$

for all $\phi \in C_{c}^{\infty}\left(\mathbb{R}^{d}\right)$. Besides, from Lemma 5.10,

$$
\left\|\left(\phi-\phi_{B}\right)\left(b-b_{B}\right) T a\right\|_{L^{1}} \leq C\|b\|_{\mathrm{BMO}}\|\phi\|_{\mathrm{BMO}} \leq C\|b\|_{\mathrm{BMO}_{L}^{\log }\|\phi\|_{\mathrm{BMO}_{L}} .}
$$

This together with Lemma 2 of [15] allow us to reduce (5.4) to showing that

$$
\log \left(e+\frac{\rho\left(x_{0}\right)}{r}\right)\left\|\left(b-b_{B}\right) T a\right\|_{L^{1}} \leq C\|b\|_{\mathrm{BMO}_{L}^{\log } .}
$$


Setting $\varepsilon=\delta / 2$, it is easy to check that there exists a constant $C=C(\varepsilon)>0$ such that

$$
\log (e+k t) \leq C k^{\varepsilon} \log (e+t)
$$

for all $k \geq 2, t>0$. Consequently, for all $k \geq 1$,

$$
\log \left(e+\frac{\rho\left(x_{0}\right)}{r}\right) \leq C 2^{k \varepsilon} \log \left(e+\left(\frac{\rho\left(x_{0}\right)}{2^{k+1} r}\right)^{k_{0}+1}\right) .
$$

Then, by Lemma 4.4 and Lemma 5.9, we get

$$
\begin{aligned}
\log (e+ & \left.\frac{\rho\left(x_{0}\right)}{r}\right)\left\|\left(b-b_{B}\right) T a\right\|_{L^{1}} \\
= & \log \left(e+\frac{\rho\left(x_{0}\right)}{r}\right)\left\|\left(b-b_{B}\right) T a\right\|_{L^{1}(2 B)} \\
& +\sum_{k \geq 1} \log \left(e+\frac{\rho\left(x_{0}\right)}{r}\right)\left\|\left(b-b_{B}\right) T a\right\|_{L^{1}\left(2^{k+1} B \backslash 2^{k} B\right)} \\
\leq & C \log \left(e+\left(\frac{\rho\left(x_{0}\right)}{2 r}\right)^{k_{0}+1}\right)\left\|b-b_{B}\right\|_{L^{2}(2 B)}\|T a\|_{L^{2}} \\
& +C \sum_{k \geq 1} 2^{k \varepsilon} \log \left(e+\left(\frac{\rho\left(x_{0}\right)}{2^{k+1} r}\right)^{k_{0}+1}\right)\left\|b-b_{B}\right\|_{L^{2}\left(2^{k+1} B\right)}\|T a\|_{L^{2}\left(2^{k+1} B \backslash 2^{k} B\right)} \\
\leq & C|2 B|^{1 / 2}\|b\|_{\mathrm{BMO}}{ }_{L}^{\log }\|a\|_{L^{2}} \\
& +C \sum_{k \geq 1} 2^{k \varepsilon}(k+1)\left|2^{k+1} B\right|^{1 / 2}\|b\|_{\mathrm{BMO}_{L}^{\log }} 2^{-k \delta}\left|2^{k} B\right|^{-1 / 2} \\
\leq & C\|b\|_{\mathrm{BMO}}^{\log ,}
\end{aligned}
$$

where we used $\delta=2 \varepsilon$. This ends the proof of (i).

(ii) By Remark 2.10, (ii) can be seen as a consequence of Theorem 3.6 that we are going to prove now.

Next, let us recall the following lemma due to Tang and Bi [44].

Lemma 5.11 (see [44], Lemma 3.1). Let $V \in R H_{d / 2}$. Then, there exists $c_{0} \in(0,1)$ such that for any positive number $N$ and $0<h<|x-y| / 16$, we have

$$
\left|K_{j}(x, y)\right| \leq \frac{C(N)}{\left(1+\frac{|x-y|}{\rho(y)}\right)^{N}} \frac{1}{|x-y|^{d-1}}\left(\int_{B(x,|x-y|)} \frac{V(z)}{|x-z|^{d-1}} d z+\frac{1}{|x-y|}\right)
$$

and

$$
\begin{aligned}
\mid K_{j}(x, y & +h)-K_{j}(x, y) \mid \\
& \leq \frac{C(N)}{\left(1+\frac{|x-y|}{\rho(y)}\right)^{N}} \frac{h^{c_{0}}}{|x-y|^{c_{0}+d-1}}\left(\int_{B(x,|x-y|)} \frac{V(z)}{|x-z|^{d-1}} d z+\frac{1}{|x-y|}\right),
\end{aligned}
$$

where $K_{j}(x, y), j=1, \ldots, d$, are the kernels of the Riesz transforms $R_{j}$. 
In order to prove Theorem 3.6, we need also the following two technical lemmas, whose proofs will be given in Section 6 .

Lemma 5.12. Let $1<q \leq d / 2$ and $c_{0}$ be as in Lemma 5.11. Then, $R_{j}(a)$ is $C$ times a classical $\left(H^{1}, q, c_{0}\right)$-molecule (e.g. [40]) for all generalized $\left(H_{L}^{1}, q, c_{0}\right)$-atom a related to the ball $B=B\left(x_{0}, r\right)$. Furthermore, for any $N>0$ and $k \geq 4$, we have

$$
\left\|R_{j}(a)\right\|_{L^{q}\left(2^{k+1} B \backslash 2^{k} B\right)} \leq \frac{C(N)}{\left(1+\frac{2^{k} r}{\rho\left(x_{0}\right)}\right)^{N}} 2^{-k c_{0}}\left|2^{k} B\right|^{1 / q-1},
$$

where $C(N)>0$ depends only on $N$.

Lemma 5.13. Let $1<q \leq d / 2$ and $\theta \geq 0$. Then, for every $f \in \operatorname{BMO}\left(\mathbb{R}^{d}\right)$, $g \in \mathrm{BMO}_{L, \theta}\left(\mathbb{R}^{d}\right)$ and $\left(H_{L}^{1}, q\right)$-atom a related to the ball $B=B\left(x_{0}, r\right)$, we have

$$
\left\|\left(g-g_{B}\right) R_{j}(a)\right\|_{L^{1}} \leq C\|g\|_{\mathrm{BMO}_{L, \theta}}
$$

and

$$
\left\|\left(f-f_{B}\right)\left(g-g_{B}\right) R_{j}(a)\right\|_{L^{1}} \leq C\|f\|_{\mathrm{BMO}}\|g\|_{\mathrm{BMO}_{L, \theta}} .
$$

Proof of Theorem 3.6. Suppose that $b \in \mathrm{BMO}_{L, \infty}^{\log }\left(\mathbb{R}^{d}\right)$, i.e. $b \in \mathrm{BMO}_{L, \theta}^{\log }\left(\mathbb{R}^{d}\right)$ for some $\theta \geq 0$. By Proposition 3.2 of [47], in order to prove that $\left[b, R_{j}\right]$ are bounded on $H_{L}^{1}\left(\mathbb{R}^{d}\right)$, it is sufficient to show that $\left\|\left[b, R_{j}\right](a)\right\|_{H_{L}^{1}} \leq C\|b\|_{\mathrm{BMO}_{L, \theta}^{\log }}$ for all $\left(H_{L}^{1}, d / 2\right)$-atom $a$. Similarly to the proof of Theorem 3.5, it remains to show

$$
\left\|\left(b-b_{B}\right) a\right\|_{H_{L}^{1}} \leq C\|b\|_{\mathrm{BMO}_{L, \theta}^{\log }}
$$

and

$$
\left\|\left(b-b_{B}\right) R_{j}(a)\right\|_{H_{L}^{1}} \leq C\|b\|_{\mathrm{BMO}_{L, \theta}^{\log }}
$$

hold for every $\left(H_{L}^{1}, d / 2\right)$-atom a related to the ball $B=B\left(x_{0}, r\right)$, where the constants $C$ in (5.8) and (5.9) are independent of $b, a$.

As before, we leave the proof of (5.8) to the interested reader.

Let us now establish (5.9). Similarly to the proof of Theorem 3.5, Lemma 5.13 allows to reduce (5.9) to showing that

$$
\log \left(e+\frac{\rho\left(x_{0}\right)}{r}\right)\left\|\left(b-b_{B}\right) R_{j}(a)\right\|_{L^{1}} \leq C\|b\|_{\mathrm{BMO}_{L, \theta}^{\log }} .
$$

Setting $\varepsilon=c_{0} / 2$, there is a constant $C=C(\varepsilon)>0$ such that for all $k \geq 1$,

$$
\log \left(e+\frac{\rho\left(x_{0}\right)}{r}\right) \leq C 2^{k \varepsilon} \log \left(e+\left(\frac{\rho\left(x_{0}\right)}{2^{k+1} r}\right)^{k_{0}+1}\right) .
$$


Note that $r \leq \mathcal{C}_{L} \rho\left(x_{0}\right)$ since $a$ is a $\left(H_{L}^{1}, d / 2\right)$-atom related to the ball $B\left(x_{0}, r\right)$. In (5.7) of Lemma 5.12, we choose $N=\left(k_{0}+1\right) \theta$. Then, Hölder inequality, (5.11) and Lemma 5.9 allow to conclude that

$$
\begin{aligned}
& \log \left(e+\frac{\rho\left(x_{0}\right)}{r}\right)\left\|\left(b-b_{B}\right) R_{j}(a)\right\|_{L^{1}} \\
= & \log \left(e+\frac{\rho\left(x_{0}\right)}{r}\right)\left\|\left(b-b_{B}\right) R_{j}(a)\right\|_{L^{1}\left(2^{4} B\right)} \\
& +\sum_{k \geq 4} \log \left(e+\frac{\rho\left(x_{0}\right)}{r}\right)\left\|\left(b-b_{B}\right) R_{j}(a)\right\|_{L^{1}\left(2^{k+1} B \backslash 2^{k} B\right)} \\
\leq & C \log \left(e+\left(\frac{\rho\left(x_{0}\right)}{2^{4} r}\right)^{k_{0}+1}\right)\left\|b-b_{B}\right\|_{L^{\frac{d}{d-2}}\left(2^{4} B\right)}\left\|R_{j}(a)\right\|_{L^{d / 2}} \\
& +C \sum_{k \geq 4} 2^{k \varepsilon} \log \left(e+\left(\frac{\rho\left(x_{0}\right)}{2^{k+1} r}\right)^{k_{0}+1}\right)\left\|b-b_{B}\right\|_{L^{\frac{d}{d-2}}\left(2^{k+1} B\right)}\left\|R_{j}(a)\right\|_{L^{d / 2}\left(2^{k+1} B \backslash 2^{k} B\right)} \\
\leq & C\|b\|_{\mathrm{BMO}_{L, \theta}^{\log }}+C\|b\|_{\mathrm{BMO}_{L, \theta}^{\log }} \sum_{k \geq 4} k 2^{-k \varepsilon} \\
\leq & C\|b\|_{\mathrm{BMO}_{L, \theta}^{\log }}
\end{aligned}
$$

where we used $c_{0}=2 \varepsilon$. This proves (5.10), and thus $\left[b, R_{j}\right]$ are bounded on $H_{L}^{1}\left(\mathbb{R}^{d}\right)$.

Conversely, assume that $\left[b, R_{j}\right]$ are bounded on $H_{L}^{1}\left(\mathbb{R}^{d}\right)$. Then, although $b$ belongs to $\mathrm{BMO}_{L, \infty}^{\log }\left(\mathbb{R}^{d}\right)$ from a duality argument and Theorem 2 of [7], we would also like to give a direct proof for completeness.

As $b \in \mathrm{BMO}_{L, \infty}\left(\mathbb{R}^{d}\right)$ by assumption, there exist $\theta \geq 0$ such that $b \in \mathrm{BMO}_{L, \theta}\left(\mathbb{R}^{d}\right)$.

For every $\left(H_{L}^{1}, d / 2\right)$-atom a related to some ball $B=B\left(x_{0}, r\right)$. By Remark 2.4 and Lemma 5.13,

$$
\begin{aligned}
\left\|R_{j}\left(\left(b-b_{B}\right) a\right)\right\|_{L^{1}} & \leq\left\|\left(b-b_{B}\right) R_{j}(a)\right\|_{L^{1}}+C\left\|\left[b, R_{j}\right](a)\right\|_{H_{L}^{1}} \\
& \leq C\|b\|_{\mathrm{BMO}_{L, \theta}}+C\left\|\left[b, R_{j}\right]\right\|_{H_{L}^{1} \rightarrow H_{L}^{1}}
\end{aligned}
$$

hold for all $j=1, \ldots, d$. In addition, noting that $r \leq \mathcal{C}_{L} \rho\left(x_{0}\right)$ since $a$ is a $\left(H_{L}^{1}, d / 2\right)$ atom related to some ball $B=B\left(x_{0}, r\right)$, Hölder inequality and Lemma 1 of [7] (see also Lemma 6.6 below) give

$$
\left\|\left(b-b_{B}\right) a\right\|_{L^{1}} \leq\left\|b-b_{B}\right\|_{L^{d /(d-2)}(B)}\|a\|_{L^{d / 2}(B)} \leq C\|b\|_{\mathrm{BMO}_{L, \theta}} .
$$

By the characterization of $H_{L}^{1}\left(\mathbb{R}^{d}\right)$ in terms of the Riesz transforms (see [16]), the above proves that $\left(b-b_{B}\right) a \in H_{L}^{1}\left(\mathbb{R}^{d}\right)$, moreover,

$$
\left\|\left(b-b_{B}\right) a\right\|_{H_{L}^{1}} \leq C\left(\|b\|_{\mathrm{BMO}_{L, \theta}}+\sum_{j=1}^{d}\left\|\left[b, R_{j}\right]\right\|_{H_{L}^{1} \rightarrow H_{L}^{1}}\right)
$$

where the constant $C>0$ is independent of $b, a$. 
Now, we prove that $b \in \mathrm{BMO}_{L, \theta}^{\log }\left(\mathbb{R}^{d}\right)$. More precisely, the following:

$$
\frac{\log \left(e+\rho\left(x_{0}\right) / r\right)}{\left(1+r / \rho\left(x_{0}\right)\right)^{\theta}} \mathrm{MO}\left(b, B\left(x_{0}, r\right)\right) \leq C\left(\|b\|_{\mathrm{BMO}_{L, \theta}}+\sum_{j=1}^{d}\left\|\left[b, R_{j}\right]\right\|_{H_{L}^{1} \rightarrow H_{L}^{1}}\right)
$$

holds for any ball $B\left(x_{0}, r\right)$ in $\mathbb{R}^{d}$. In fact, we only need to establish (5.13) for $0<r<\rho\left(x_{0}\right) / 2$ since $b \in \mathrm{BMO}_{L, \theta}\left(\mathbb{R}^{d}\right)$.

Indeed, in (5.12) we choose $B=B\left(x_{0}, r\right)$ and $a=(2|B|)^{-1}\left(f-f_{B}\right) \chi_{B}$, where $f=\operatorname{sign}\left(b-b_{B}\right)$. Then, it is easy to see that $a$ is a $\left(H_{L}^{1}, d / 2\right)$-atom related to the ball $B$. We next consider

$$
g_{x_{0}, r}(x)=\chi_{[0, r]}\left(\left|x-x_{0}\right|\right) \log \left(\frac{\rho\left(x_{0}\right)}{r}\right)+\chi_{\left(r, \rho\left(x_{0}\right)\right]}\left(\left|x-x_{0}\right|\right) \log \left(\frac{\rho\left(x_{0}\right)}{\left|x-x_{0}\right|}\right) .
$$

Then, thanks to Lemma 2.5 of [33], one has $\left\|g_{x_{0}, r}\right\|_{\mathrm{BMO}_{L}} \leq C$. Moreover, it is clear that $g_{x_{0}, r}\left(b-b_{B}\right) a \in L^{1}\left(\mathbb{R}^{d}\right)$. Consequently, (5.12) together with the fact that $\mathrm{BMO}_{L}\left(\mathbb{R}^{d}\right)$ is the dual of $H_{L}^{1}\left(\mathbb{R}^{d}\right)$ allows us to conclude that

$$
\begin{aligned}
& \frac{\log \left(e+\rho\left(x_{0}\right) / r\right)}{\left(1+r / \rho\left(x_{0}\right)\right)^{\theta}} \operatorname{MO}\left(b, B\left(x_{0}, r\right)\right) \leq 3 \log \left(\frac{\rho\left(x_{0}\right)}{r}\right) \operatorname{MO}\left(b, B\left(x_{0}, r\right)\right) \\
& =6\left|\int_{\mathbb{R}^{d}} g_{x_{0}, r}(x)\left(b(x)-b_{B}\right) a(x) d x\right| \leq 6\left\|g_{x_{0}, r}\right\|_{\mathrm{BMO}_{L}}\left\|\left(b-b_{B}\right) a\right\|_{H_{L}^{1}} \\
& \leq C\left(\|b\|_{\mathrm{BMO}_{L, \theta}}+\sum_{j=1}^{d}\left\|\left[b, R_{j}\right]\right\|_{H_{L}^{1} \rightarrow H_{L}^{1}}\right),
\end{aligned}
$$

where we used $r<\rho\left(x_{0}\right) / 2$ and

$$
\int_{\mathbb{R}^{d}}\left(b(x)-b_{B}\right) a(x) d x=\frac{1}{2\left|B\left(x_{0}, r\right)\right|} \int_{B\left(x_{0}, r\right)}\left|b(x)-b_{B\left(x_{0}, r\right)}\right| d x .
$$

This ends the proof.

\section{Proof of the key lemmas}

First, let us recall some notations and results due to Dziubański and Zienkiewicz in [16]. These notations and results play an important role in our proofs.

Let $P(x)=(4 \pi)^{-d / 2} e^{-|x|^{2} / 4}$ be the Gauss function. For $n \in \mathbb{Z}$, the space $h_{n}^{1}\left(\mathbb{R}^{d}\right)$ denotes the space of all integrable functions $f$ such that

$$
\mathcal{M}_{n} f(x)=\sup _{0<t<2^{-n}}\left|P_{\sqrt{t}} * f(x)\right|=\sup _{0<t<2^{-n}}\left|\int_{\mathbb{R}^{d}} p_{t}(x, y) f(y) d y\right| \in L^{1}\left(\mathbb{R}^{d}\right),
$$

where the kernel $p_{t}$ is given by $p_{t}(x, y)=(4 \pi t)^{-d / 2} e^{-\frac{|x-y|^{2}}{4 t}}$. We equipped this space with the norm $\|f\|_{h_{n}^{1}}:=\left\|\mathcal{M}_{n} f\right\|_{L^{1}}$.

For convenience of the reader, we list here some lemmas used in our proofs. 
Lemma 6.1 (see [16], Lemma 2.3). There exist a constant $C>0$ and a collection of balls $B_{n, k}=B\left(x_{n, k}, 2^{-n / 2}\right), n \in \mathbb{Z}, k=1,2, \ldots$, such that $x_{n, k} \in \mathcal{B}_{n}, \mathcal{B}_{n} \subset$ $\bigcup_{k} B_{n, k}$, and

$$
\operatorname{card}\left\{\left(n^{\prime}, k^{\prime}\right): B\left(x_{n, k}, R 2^{-n / 2}\right) \cap B\left(x_{n^{\prime}, k^{\prime}}, R 2^{-n / 2}\right) \neq \emptyset\right\} \leq R^{C}
$$

for all $n, k$ and $R \geq 2$.

Lemma 6.2 (see [16], Lemma 2.5). There are nonnegative $C^{\infty}$-functions $\psi_{n, k}$, $n \in \mathbb{Z}, k=1,2, \ldots$, supported in the balls $B\left(x_{n, k}, 2^{1-n / 2}\right)$, such that

$$
\sum_{n, k} \psi_{n, k}=1 \quad \text { and } \quad\left\|\nabla \psi_{n, k}\right\|_{L^{\infty}} \leq C 2^{n / 2}
$$

Lemma 6.3 (see (4.7) in [16]). For every $f \in H_{L}^{1}\left(\mathbb{R}^{d}\right)$, we have

$$
\sum_{n, k}\left\|\psi_{n, k} f\right\|_{h_{n}^{1}} \leq C\|f\|_{H_{L}^{1}}
$$

To prove Lemma 5.4, we need the following.

Lemma 6.4. There exists a constant $C=C(\varphi, d)>0$ such that

$$
\left\|f-\varphi_{2^{-n / 2}} * f\right\|_{H^{1}} \leq C\|f\|_{h_{n}^{1}}, \quad \text { for all } n \in \mathbb{Z}, f \in h_{n}^{1}\left(\mathbb{R}^{d}\right) .
$$

The proof of Lemma 6.4 can be found in [20]. In fact, in [20], Goldberg proved it just for $n=0$; however, by dilations, it is easy to see that (6.1) holds for every $n \in \mathbb{Z}, f \in h_{n}^{1}\left(\mathbb{R}^{d}\right)$ with an uniform constant $C>0$ depends only on $\varphi$ and $d$.

Proof of Lemma 5.4. It follows from Lemma 6.4 and Lemma 6.3 that

$$
\begin{aligned}
\|\mathfrak{H}(f)\|_{H^{1}} & =\left\|\sum_{n, k}\left(\psi_{n, k} f-\varphi_{2^{-n / 2}} *\left(\psi_{n, k} f\right)\right)\right\|_{H^{1}} \\
& \leq \sum_{n, k}\left\|\psi_{n, k} f-\varphi_{2^{-n / 2}} *\left(\psi_{n, k} f\right)\right\|_{H^{1}} \leq C \sum_{n, k}\left\|\psi_{n, k} f\right\|_{h_{n}^{1}} \leq C\|f\|_{H_{L}^{1}}
\end{aligned}
$$

for every $f \in H_{L}^{1}\left(\mathbb{R}^{d}\right)$. This completes the proof.

For $1<q \leq \infty$ and $n \in \mathbb{Z}$. Recall (see [16]) that a function $a$ is said to be a $\left(h_{n}^{1}, q\right)$-atom related to the ball $B\left(x_{0}, r\right)$ if $r \leq 2^{1-n / 2}$ and

(i) supp $a \subset B\left(x_{0}, r\right)$,

(ii) $\|a\|_{L^{q}} \leq\left|B\left(x_{0}, r\right)\right|^{1 / q-1}$,

(iii) if $r \leq 2^{-1-n / 2}$ then $\int_{\mathbb{R}^{d}} a(x) d x=0$.

In order to prove Lemma 5.5, we need the following lemma. 
Lemma 6.5. Let $1<q \leq \infty, n \in \mathbb{Z}$ and $x \in \mathcal{B}_{n}$. Suppose that $f \in h_{n}^{1}\left(\mathbb{R}^{d}\right)$ with supp $f \subset B\left(x, 2^{1-n / 2}\right)$. Then, there are $\left(H_{L}^{1}, q\right)$-atoms $a_{j}$ related to the balls $B\left(x_{j}, r_{j}\right)$ such that $B\left(x_{j}, r_{j}\right) \subset B\left(x, 2^{2-n / 2}\right)$ and

$$
f=\sum_{j} \lambda_{j} a_{j}, \quad \sum_{j}\left|\lambda_{j}\right| \leq C\|f\|_{h_{n}^{1}}
$$

with a positive constant $C$ independent of $n$ and $f$.

Proof. By Theorem 4.5 of [16], there are $\left(h_{n}^{1}, q\right)$-atoms $a_{j}$ related to the balls $B\left(x_{j}, r_{j}\right)$ such that $B\left(x_{j}, r_{j}\right) \subset B\left(x, 2^{2-n / 2}\right)$ and

$$
f=\sum_{j} \lambda_{j} a_{j}, \quad \sum_{j}\left|\lambda_{j}\right| \leq C\|f\|_{h_{n}^{1}} .
$$

Now, let us establish that the $a_{j}$ 's are $\left(H_{L}^{1}, q\right)$-atoms related to the balls $B\left(x_{j}, r_{j}\right)$.

Indeed, as $x_{j} \in B\left(x, 2^{2-n / 2}\right)$ and $x \in \mathcal{B}_{n}$, Proposition 2.1 implies that $r_{j} \leq$ $2^{2-n / 2} \leq \mathcal{C}_{L} \rho\left(x_{j}\right)$, where $\mathcal{C}_{L}$ is as in (2.4). Moreover, if $r_{j}<\frac{1}{\mathcal{C}_{L}} \rho\left(x_{j}\right)$, then Proposition 2.1 implies that $r_{j} \leq 2^{-1-n / 2}$, and thus $\int_{\mathbb{R}^{d}} a_{j}(x) d x=0$ since $a_{j}$ are $\left(h_{n}^{1}, q\right)$-atoms related to the balls $B\left(x_{j}, r_{j}\right)$. These prove that the $a_{j}$ 's are $\left(H_{L}^{1}, q\right)$-atoms related to the balls $B\left(x_{j}, r_{j}\right)$.

Proof of Lemma 5.5. As $T \in \mathcal{K}_{L}$, there exist $q \in(1, \infty]$ and $\varepsilon>0$ such that

$$
\left\|\left(b-b_{B}\right) T a\right\|_{L^{1}} \leq C\|b\|_{\mathrm{BMO}}
$$

for all $b \in \operatorname{BMO}\left(\mathbb{R}^{d}\right)$ and generalized $\left(H_{L}^{1}, q, \varepsilon\right)$-atom $a$ related to the ball $B$.

From the fact that $\mathbb{H}_{L, \text {,in }}^{1, q, \varepsilon}\left(\mathbb{R}^{d}\right)$ is dense in $\mathbb{H}_{L, \text { at }}^{1, q, \varepsilon}\left(\mathbb{R}^{d}\right)=H_{L}^{1}\left(\mathbb{R}^{d}\right.$ ) (see Theorem 2.5), we need only prove that

$$
\|\mathcal{U}(f, b)\|_{L^{1}}=\|[b, T](f-\mathfrak{H}(f))\|_{L^{1}} \leq C\|f\|_{H_{L}^{1}}\|b\|_{\mathrm{BMO}}
$$

holds for every $(f, b) \in \mathbb{H}_{L, \text { in }}^{1, q, \varepsilon}\left(\mathbb{R}^{d}\right) \times \operatorname{BMO}\left(\mathbb{R}^{d}\right)$.

For any $(n, k) \in \mathbb{Z} \times \mathbb{Z}^{+}$. As $x_{n, k} \in \mathcal{B}_{n}$ and $\psi_{n, k} f \in h_{n}^{1}\left(\mathbb{R}^{d}\right)$, it follows from Lemma 6.5 and Remark 2.4 that there are generalized $\left(H_{L}^{1}, q, \varepsilon\right)$-atoms $a_{j}^{n, k}$ related to the balls $B\left(x_{j}^{n, k}, r_{j}^{n, k}\right)$ such that $B\left(x_{j}^{n, k}, r_{j}^{n, k}\right) \subset B\left(x_{n, k}, 2^{2-n / 2}\right)$ and

$$
\psi_{n, k} f=\sum_{j} \lambda_{j}^{n, k} a_{j}^{n, k}, \quad \sum_{j}\left|\lambda_{j}^{n, k}\right| \leq C\left\|\psi_{n, k} f\right\|_{h_{n}^{1}}
$$

with a positive constant $C$ independent of $n, k$ and $f$.

Clearly, supp $\varphi_{2^{-n / 2}} * a_{j}^{n, k} \subset B\left(x_{n, k}, 5.2^{-n / 2}\right)$ since supp $\varphi \subset B(0,1)$ and supp $a_{j}^{n, k} \subset B\left(x_{n, k}, 2^{2-n / 2}\right)$; the following estimate holds:

$$
\begin{aligned}
\left\|\varphi_{2^{-n / 2}} * a_{j}^{n, k}\right\|_{L^{q}} & \leq\left\|\varphi_{2^{-n / 2}}\right\|_{L^{q}}\left\|a_{j}^{n, k}\right\|_{L^{1}} \leq\left(2^{-n / 2}\right)^{d(1 / q-1)}\|\varphi\|_{L^{q}} \\
& \leq C\left|B\left(x_{n, k}, 5.2^{-n / 2}\right)\right|^{1 / q-1} .
\end{aligned}
$$


Moreover, as $x_{n, k} \in \mathcal{B}_{n}$,

$$
\left|\int_{\mathbb{R}^{d}} \varphi_{2^{-n / 2}} * a_{j}^{n, k} d x\right| \leq\left\|\varphi_{2^{-n / 2}}\right\|_{L^{1}}\left\|a_{j}^{n, k}\right\|_{L^{1}} \leq C\left(\frac{5.2^{-n / 2}}{\rho\left(x_{n, k}\right)}\right)^{\varepsilon} .
$$

These prove that $\varphi_{2^{-n / 2}} * a_{j}^{n, k}$ is $C$ times a generalized $\left(H_{L}^{1}, q, \varepsilon\right)$-atom related to $B\left(x_{n, k}, 5.2^{-n / 2}\right)$. Consequently, (6.2) yields

$$
\left\|\left(b-b_{B\left(x_{n, k}, 5.2^{-n / 2}\right)}\right) T\left(\varphi_{2^{-n / 2}} * a_{j}^{n, k}\right)\right\|_{L^{1}} \leq C\|b\|_{\mathrm{BMO}} .
$$

By an analogous argument, it is easy to check that

$$
\left(\varphi_{2-n / 2} * a_{j}^{n, k}\right)\left(b-b_{B\left(x_{n, k}, 5.2^{-n / 2}\right)}\right)
$$

is $C\|b\|_{\text {BMo }}$ times a generalized $\left(H_{L}^{1}, \frac{q+1}{2}, \varepsilon\right)$-atom related to $B\left(x_{n, k}, 5.2^{-n / 2}\right)$. Hence, it follows from (6.3) and (6.4) that

$$
\begin{aligned}
\left\|[b, T]\left(\varphi_{2^{-n / 2}} *\left(\psi_{n, k} f\right)\right)\right\|_{L^{1}} \leq & \left\|\left(b-b_{B\left(x_{n, k}, 5.2^{-n / 2}\right)}\right) T\left(\varphi_{2^{-n / 2}} *\left(\psi_{n, k} f\right)\right)\right\|_{L^{1}} \\
& +\left\|T\left(\left(b-b_{B\left(x_{n, k}, 5.2^{-n / 2}\right)}\right)\left(\varphi_{2^{-n / 2}} *\left(\psi_{n, k} f\right)\right)\right)\right\|_{L^{1}} \\
\leq & C\left\|\psi_{n, k} f\right\|_{h_{n}^{1}}\|b\|_{\mathrm{BMO}},
\end{aligned}
$$

where we used the fact that $T$ is bounded from $H_{L}^{1}\left(\mathbb{R}^{d}\right)$ into $L^{1}\left(\mathbb{R}^{d}\right)$ since $T \in \mathcal{K}_{L}$.

On the other hand, by $f \in \mathbb{H}_{L, \text { fin }}^{1, q, \varepsilon}\left(\mathbb{R}^{d}\right)$, there exists a ball $B(0, R)$ such that supp $f \subset B(0, R)$. As $\overline{B(0, R)}$ is a compact set, Lemma 6.1 allows to conclude that there is a finite set $\Gamma_{R} \subset \mathbb{Z} \times \mathbb{Z}^{+}$such that for every $(n, k) \notin \Gamma_{R}$,

$$
B\left(x_{n, k}, 2^{1-n / 2}\right) \cap \overline{B(0, R)}=\emptyset .
$$

It follows that there are $N, K \in \mathbb{Z}^{+}$such that

$$
f=\sum_{n, k} \psi_{n, k} f=\sum_{n=-N}^{N} \sum_{k=1}^{K} \psi_{n, k} f .
$$

Therefore, (6.5) and Lemma 6.3 yield

$$
\begin{aligned}
\|\mathcal{U}(f, b)\|_{L^{1}} & \leq\left\|\sum_{n=-N}^{N} \sum_{k=1}^{K}\left|[b, T]\left(\varphi_{2^{-n / 2}} *\left(\psi_{n, k} f\right)\right)\right|\right\|_{L^{1}} \\
& \leq C\|b\|_{\mathrm{BMO}} \sum_{n, k}\left\|\psi_{n, k} f\right\|_{h_{n}^{1}} \leq C\|f\|_{H_{L}^{1}}\|b\|_{\mathrm{BMO}},
\end{aligned}
$$

which ends the proof.

Proof of Lemma 5.9. First, we claim that for every ball $B_{0}=B\left(x_{0}, r_{0}\right)$,

$$
\left(\frac{1}{\left|B_{0}\right|} \int_{B_{0}}\left|f(y)-f_{B_{0}}\right|^{q} d y\right)^{1 / q} \leq C \frac{\left(1+\frac{r_{0}}{\rho\left(x_{0}\right)}\right)^{\left(k_{0}+1\right) \theta}}{\log \left(e+\left(\frac{\rho\left(x_{0}\right)}{r_{0}}\right)^{k_{0}+1}\right)}\|f\|_{\mathrm{BMO}_{L, \theta}^{\log } .} .
$$


Assume that (6.6) holds for a moment. Then,

$$
\begin{aligned}
& \left(\frac{1}{\left|2^{k} B\right|} \int_{2^{k} B}\left|f(y)-f_{B}\right|^{q} d y\right)^{1 / q} \\
& \quad \leq\left(\frac{1}{\left|2^{k} B\right|} \int_{2^{k} B}\left|f(y)-f_{2^{k} B}\right|^{q} d y\right)^{1 / q}+\sum_{j=0}^{k-1}\left|f_{2^{j+1} B}-f_{2^{j} B}\right| \\
& \quad \leq \frac{\left(1+\frac{2^{k} r}{\rho(x)}\right)^{\left(k_{0}+1\right) \theta}}{\log \left(e+\left(\frac{\rho(x)}{2^{k} r}\right)^{k_{0}+1}\right)}\|f\|_{\mathrm{BMO}_{L, \theta}^{\log }}+\sum_{j=0}^{k-1} 2^{d} \frac{\left(1+\frac{2^{j+1} r}{\rho(x)}\right)^{\theta}}{\log \left(e+\frac{\rho(x)}{2^{j+1} r}\right)}\|f\|_{\mathrm{BMO}_{L, \theta}^{\log }} \\
& \quad \leq C k \frac{\left(1+\frac{2^{k} r}{\rho(x)}\right)^{\left(k_{0}+1\right) \theta}}{\log \left(e+\left(\frac{\rho(x)}{2^{k} r}\right)^{k_{0}+1}\right)}\|f\|_{\mathrm{BMO}_{L, \theta}^{\log } \cdot}
\end{aligned}
$$

It remains to prove (6.6). Let us define the function $h$ on $\mathbb{R}^{d}$ as follows:

$$
h(x)= \begin{cases}1, & x \in B_{0}, \\ \frac{2 r_{0}-\left|x-x_{0}\right|}{r_{0}}, & x \in 2 B_{0} \backslash B_{0}, \\ 0, & x \notin 2 B_{0},\end{cases}
$$

and notice that

$$
|h(x)-h(y)| \leq \frac{|x-y|}{r_{0}} .
$$

Setting $\tilde{f}:=f-f_{2 B_{0}}$. By the classical John-Nirenberg inequality, there exists a constant $C=C(d, q)>0$ such that

$$
\begin{aligned}
\left(\frac{1}{\left|B_{0}\right|} \int_{B_{0}}\left|f(y)-f_{B_{0}}\right|^{q} d y\right)^{1 / q} & =\left(\frac{1}{\left|B_{0}\right|} \int_{B_{0}}\left|h(y) \widetilde{f}(y)-(h \widetilde{f})_{B_{0}}\right|^{q} d y\right)^{1 / q} \\
& \leq C\|h \widetilde{f}\|_{\text {BMO }}
\end{aligned}
$$

Therefore, the proof of the lemma is reduced to showing that

$$
\|h \widetilde{f}\|_{\mathrm{BMO}} \leq C \frac{\left(1+r_{0} / \rho\left(x_{0}\right)\right)^{\left(k_{0}+1\right) \theta}}{\log \left(e+\left(\rho\left(x_{0}\right) / r_{0}\right)^{k_{0}+1}\right)}\|f\|_{\mathrm{BMO}_{L, \theta}^{\log },}
$$

namely, for every ball $B=B(x, r)$,

$$
\frac{1}{|B|} \int_{B}\left|h(y) \tilde{f}(y)-(h \tilde{f})_{B}\right| d y \leq C \frac{\left(1+r_{0} / \rho\left(x_{0}\right)\right)^{\left(k_{0}+1\right) \theta}}{\log \left(e+\left(\rho\left(x_{0}\right) / r_{0}\right)^{k_{0}+1}\right)}\|f\|_{\mathrm{BMO}_{L, \theta}^{\log }} .
$$

Now, let us focus on inequality (6.8). Noting that supp $h \subset 2 B_{0}$, inequality (6.8) is obvious if $B \cap 2 B_{0}=\emptyset$. Hence, we only consider the case $B \cap 2 B_{0} \neq \emptyset$. Then, we have the following two cases: 
The case $r>r_{0}$. The fact $B \cap 2 B_{0} \neq \emptyset$ implies that $2 B_{0} \subset 5 B$, and thus

$$
\begin{aligned}
& \frac{1}{|B|} \int_{B}\left|h(y) \widetilde{f}(y)-(h \widetilde{f})_{B}\right| d y \leq 2 \frac{1}{|B|} \int_{B}|h(y) \widetilde{f}(y)| d y \\
& \quad \leq 2.5^{d} \frac{1}{\left|2 B_{0}\right|} \int_{2 B_{0}}\left|f(y)-f_{2 B_{0}}\right| d y \leq C \frac{\left(1+2 r_{0} / \rho\left(x_{0}\right)\right)^{\theta}}{\log \left(e+\rho\left(x_{0}\right) /\left(2 r_{0}\right)\right)}\|f\|_{\mathrm{BMO}_{L, \theta}^{\log }} \\
& \quad \leq C \frac{\left(1+r_{0} / \rho\left(x_{0}\right)\right)^{\left(k_{0}+1\right) \theta}}{\log \left(e+\left(\rho\left(x_{0}\right) / r_{0}\right)^{k_{0}+1}\right)}\|f\|_{\mathrm{BMO}_{L, \theta}^{\log .} \cdot}
\end{aligned}
$$

The case $r \leq r_{0}$. Inequality (6.7) yields

$$
\begin{aligned}
\frac{1}{|B|} \int_{B}\left|h(y) \widetilde{f}(y)-(h \widetilde{f})_{B}\right| d y \leq & 2 \frac{1}{|B|} \int_{B}\left|h(y) \widetilde{f}(y)-h_{B} \widetilde{f}_{B}\right| d y \\
\leq & 2 \frac{1}{|B|} \int_{B}\left|h(y)\left(\widetilde{f}(y)-\widetilde{f}_{B}\right)\right| d y \\
& +2\left|\widetilde{f}_{B}\right| \frac{1}{|B|} \int_{B} \frac{1}{|B|}\left|\int_{B}(h(x)-h(y)) d y\right| d x \\
\leq & 2 \frac{1}{|B|} \int_{B}\left|f(y)-f_{B}\right| d y+4 \frac{r}{r_{0}}\left|f_{B}-f_{2 B_{0}}\right| .
\end{aligned}
$$

By $r \leq r_{0}, B=B(x, r) \cap B\left(x_{0}, r_{0}\right) \neq \emptyset$, Proposition 2.1 gives

$$
\frac{r}{\rho(x)} \leq \frac{r_{0}}{\rho(x)} \leq \kappa \frac{r_{0}}{\rho\left(x_{0}\right)}\left(1+\frac{\left|x-x_{0}\right|}{\rho\left(x_{0}\right)}\right)^{k_{0}} \leq C\left(1+\frac{r_{0}}{\rho\left(x_{0}\right)}\right)^{k_{0}+1} .
$$

Consequently,

$$
\begin{aligned}
\frac{1}{|B|} \int_{B}\left|f(y)-f_{B}\right| d y & \leq \frac{(1+r / \rho(x))^{\theta}}{\log (e+\rho(x) / r)}\|f\|_{\mathrm{BMO}_{L, \theta}^{\log }} \\
& \leq C \frac{\left(1+r_{0} / \rho\left(x_{0}\right)\right)^{\left(k_{0}+1\right) \theta}}{\log \left(e+\left(\rho\left(x_{0}\right) / r_{0}\right)^{k_{0}+1}\right)}\|f\|_{\mathrm{BMO}_{L, \theta}^{\log },}
\end{aligned}
$$

and

$$
\begin{aligned}
& \frac{1}{\left|B\left(x, 2^{3} r_{0}\right)\right|} \int_{B\left(x, 2^{3} r_{0}\right)}\left|f(y)-f_{B\left(x, 2^{3} r_{0}\right)}\right| d y \leq \frac{\left(1+2^{3} r_{0} / \rho(x)\right)^{\theta}}{\log \left(e+\rho(x) /\left(2^{3} r_{0}\right)\right)}\|f\|_{\mathrm{BMO}_{L, \theta}^{\log }} \\
& \leq C \frac{\left(1+r_{0} / \rho\left(x_{0}\right)\right)^{\left(k_{0}+1\right) \theta}}{\log \left(e+\left(\rho\left(x_{0}\right) / r_{0}\right)^{k_{0}+1}\right)}\|f\|_{\mathrm{BMO}_{L, \theta}^{\log }} .
\end{aligned}
$$

Noting that, for every $k \in \mathbb{N}$ with $2^{k+1} r \leq 2^{3} r_{0}$,

$$
\begin{aligned}
& \left|f_{2^{k+1} B}-f_{2^{k} B}\right| \leq 2^{d} \frac{1}{\left|2^{k+1} B\right|} \int_{2^{k+1} B}\left|f(y)-f_{2^{k+1} B}\right| d y \\
& \quad \leq C \frac{\left(1+2^{3} r_{0} / \rho(x)\right)^{\theta}}{\log \left(e+\rho(x) /\left(2^{3} r_{0}\right)\right)}\|f\|_{\mathrm{BMO}_{L, \theta}^{\log }} \leq C \frac{\left(1+r_{0} / \rho\left(x_{0}\right)\right)^{\left(k_{0}+1\right) \theta}}{\log \left(e+\left(\rho\left(x_{0}\right) / r_{0}\right)^{k_{0}+1}\right)}\|f\|_{\mathrm{BMO}_{L, \theta}^{\log },},
\end{aligned}
$$


allows us to conclude that

$$
\left|f_{B(x, r)}-f_{B\left(x, 2^{3} r_{0}\right)}\right| \leq C \log \left(e+r_{0} / r\right) \frac{\left(1+r_{0} / \rho\left(x_{0}\right)\right)^{\left(k_{0}+1\right) \theta}}{\log \left(e+\left(\rho\left(x_{0}\right) / r_{0}\right)^{k_{0}+1}\right)}\|f\|_{\mathrm{BMO}_{L, \theta}^{\log }} .
$$

Then, the inclusion $2 B_{0} \subset B\left(x, 2^{3} r_{0}\right)$, together with the inequalities (6.9), (6.10), (6.11) and (6.12), yield

$$
\begin{aligned}
\frac{1}{|B|} \int_{B} \mid h(y) \tilde{f}(y)- & (h \tilde{f})_{B}\left|d y \leq 2 \frac{1}{|B|} \int_{B}\right| f(y)-f_{B} \mid d y \\
& +4 \frac{r}{r_{0}}\left(\left|f_{B(x, r)}-f_{B\left(x, 2^{3} r_{0}\right)}\right|+4^{d} M O\left(f, B\left(x, 2^{3} r_{0}\right)\right)\right) \\
\leq & C\left(1+\frac{r}{r_{0}} \log \left(e+\frac{r_{0}}{r}\right)\right) \frac{\left(1+r_{0} / \rho\left(x_{0}\right)\right)^{\left(k_{0}+1\right) \theta}}{\log \left(e+\left(\rho\left(x_{0}\right) / r_{0}\right)^{k_{0}+1}\right)}\|f\|_{\mathrm{BMO}_{L, \theta}^{\log }} \\
\leq & C \frac{\left(1+r_{0} / \rho\left(x_{0}\right)\right)^{\left(k_{0}+1\right) \theta}}{\log \left(e+\left(\rho\left(x_{0}\right) / r_{0}\right)^{k_{0}+1}\right)}\|f\|_{\mathrm{BMO}_{L, \theta}^{\log },}
\end{aligned}
$$

where we used $\frac{r}{r_{0}} \log \left(e+\frac{r_{0}}{r}\right) \leq \sup _{t \leq 1} t \log (e+1 / t)<\infty$. This ends the proof.

By an analogous argument, we can also obtain the following, which was proved by Bongioanni et al (see Lemma 1 of [7]) through another method.

Lemma 6.6. Let $1 \leq q<\infty$ and $\theta \geq 0$. Then, for every $f \in \mathrm{BMO}_{L, \theta}\left(\mathbb{R}^{d}\right)$, $B=B(x, r)$ and $k \in \mathbb{Z}^{+}$, we have

$$
\left(\frac{1}{\left|2^{k} B\right|} \int_{2^{k} B}\left|f(y)-f_{B}\right|^{q} d y\right)^{1 / q} \leq C k\left(1+\frac{2^{k} r}{\rho(x)}\right)^{\left(k_{0}+1\right) \theta}\|f\|_{\mathrm{BMO}_{L, \theta}} .
$$

Proof of Lemma 5.10. (i) Assume that $T$ is a $(\delta, L)$-Calderón-Zygmund operator for some $\delta \in(0,1]$. For every generalized $\left(H_{L}^{1}, 2, \delta\right)$-atom $a$ related to the ball $B$, as $T^{*} 1=0$, Lemma 4.4 implies that $T a$ is $C$ times a classical $\left(H^{1}, 2, \delta\right)$-molecule (see for example [40]) related to $B$, and thus $\|T a\|_{H^{1}} \leq C$. Therefore, Proposition 2.7 yields $T$ maps continuously $H_{L}^{1}\left(\mathbb{R}^{d}\right)$ into $H^{1}\left(\mathbb{R}^{d}\right)$.

(ii) By Lemma 4.3, Lemma 4.4 and Hölder inequality, we get

$$
\begin{aligned}
\| & \left(f-f_{B}\right)\left(g-g_{B}\right) T a \|_{L^{1}} \\
= & \left\|\left(f-f_{B}\right)\left(g-g_{B}\right) T a\right\|_{L^{1}(2 B)}+\sum_{k \geq 1}\left\|\left(f-f_{B}\right)\left(g-g_{B}\right) T a\right\|_{L^{1}\left(2^{k+1} B \backslash 2^{k} B\right)} \\
\leq & \left\|f-f_{B}\right\|_{L^{2 q^{\prime}}(2 B)}\left\|g-g_{B}\right\|_{L^{2 q^{\prime}}(2 B)}\|T(a)\|_{L^{q}} \\
& +\sum_{k \geq 1}\left\|f-f_{B}\right\|_{L^{2 q^{\prime}}\left(2^{k+1} B\right)}\left\|g-g_{B}\right\|_{L^{2 q^{\prime}\left(2^{k+1} B\right)}}\|T(a)\|_{L^{q}\left(2^{k+1} B \backslash 2^{k} B\right)} \\
\leq & C\|f\|_{\mathrm{BMO}}\|g\|_{\mathrm{BMO}}+\sum_{k \geq 1} C(k+1)^{2}\|f\|_{\mathrm{BMO}}\|g\|_{\mathrm{BMO}}\left|2^{k+1} B\right|^{1 / q^{\prime}} 2^{-k \delta}\left|2^{k} B\right|^{1 / q-1} \\
\leq & C\|f\|_{\mathrm{BMO}}\|g\|_{\mathrm{BMO}},
\end{aligned}
$$

where $1 / q+1 / q^{\prime}=1$. 
Proof of Lemma 5.12. It is well-known that the Riesz transforms $R_{j}$ are bounded from $H_{L}^{1}\left(\mathbb{R}^{d}\right)$ into $H^{1}\left(\mathbb{R}^{d}\right)$, in particular, one has $\int_{\mathbb{R}^{d}} R_{j}(a)(x) d x=0$. Moreover, by the $L^{q}$-boundedness of $R_{j}$ (see [39], Theorem 0.5 ) one has $\left\|R_{j}(a)\right\|_{L^{q}} \leq$ $C|B|^{1 / q-1}$. Therefore, it is sufficient to verify (5.7). Thanks to Lemma 5.11 , as $a$ is a generalized $\left(H_{L}^{1}, q, c_{0}\right)$-atom related to the ball $B$, for every $x \in 2^{k+1} B \backslash 2^{k} B$,

$$
\begin{aligned}
& \left|R_{j}(a)(x)\right| \leq\left|\int_{B}\left(K_{j}(x, y)-K_{j}\left(x, x_{0}\right)\right) a(y) d y\right|+\left|K_{j}\left(x, x_{0}\right)\right|\left|\int_{B} a(y) d y\right| \\
& \leq \int_{B} \frac{C(N)}{\left(1+\left|x-x_{0}\right| / \rho\left(x_{0}\right)\right)^{N+4 N_{0}}} \frac{\left|y-x_{0}\right|^{c_{0}}}{\left|x-x_{0}\right|^{d+c_{0}-1}} \\
& \quad\left\{\int_{B\left(x,\left|x-x_{0}\right|\right)} \frac{V(z)}{|x-z|^{d-1}} d z+\frac{1}{\left|x-x_{0}\right|}\right\}|a(y)| d y \\
& +\frac{C(N)}{\left(1+\left|x-x_{0}\right| / \rho\left(x_{0}\right)\right)^{N+4 N_{0}+c_{0}}} \frac{1}{\left|x-x_{0}\right|^{d-1}} \\
& \quad\left(\int_{B\left(x,\left|x-x_{0}\right|\right)} \frac{V(z)}{|x-z|^{d-1}} d z+\frac{1}{\left|x-x_{0}\right|}\right)\left(\frac{r}{\rho\left(x_{0}\right)}\right)^{c_{0}}
\end{aligned}
$$

$$
\leq \frac{C(N)}{\left(1+\frac{2^{k} r}{\rho\left(x_{0}\right)}\right)^{N}}\left(\frac{1}{\left(1+\frac{2^{k+2} r}{\rho\left(x_{0}\right)}\right)^{N_{0}}} \frac{r^{c_{0}}}{\left(2^{k} r\right)^{d+c_{0}-1}} \cdot \int_{B\left(x,\left|x-x_{0}\right|\right)} \frac{V(z)}{|x-z|^{d-1}} d z+\frac{2^{-k c_{0}}}{\left|2^{k} B\right|}\right) .
$$

Here and in what follows, the constants $C(N)$ depend only on $N$, but may change from line to line. Note that for every $x \in 2^{k+1} B \backslash 2^{k} B, B\left(x,\left|x-x_{0}\right|\right) \subset B\left(x, 2^{k+1} r\right)$ $\subset B\left(x_{0}, 2^{k+2} r\right)$. The fact $V \in R H_{d / 2}, d / 2 \geq q>1$, and Hölder's inequality yield

$$
\begin{aligned}
& \text { 14) }\left\|\int_{B\left(x,\left|x-x_{0}\right|\right)} \frac{V(z)}{|x-z|^{d-1}} d z\right\|_{L^{q}\left(2^{k+1} B \backslash 2^{k} B, d x\right)} \\
& \leq C\left(2^{k+1} r\right)^{1-2 / d}\left\{\int_{2^{k+1} B \backslash 2^{k} B}\left(\int_{B\left(x, 2^{k+1} r\right)} \frac{|V(z)|^{d / 2}}{|x-z|^{d-1}} d z\right)^{2 q / d} d x\right\}^{1 / q} \\
& \leq C\left(2^{k} r\right)^{1-2 / d}\left|2^{k+1} B\right|^{1 / q-2 / d}\left\{\int_{B\left(z, 2^{k+1} r\right)} d x \int_{B\left(x_{0}, 2^{k+2} r\right)} \frac{|V(z)|^{d / 2}}{|x-z|^{d-1}} d z\right\}^{2 / d} \\
& \leq C 2^{k} r\left|2^{k} B\right|^{1 / q-1} \int_{B\left(x_{0}, 2^{k+2} r\right)} V(z) d z .
\end{aligned}
$$

Combining (6.13), (6.14) and Lemma 1 of [21], we obtain that

$$
\begin{aligned}
\left\|R_{j}(a)\right\|_{L^{q}\left(2^{k+1} B \backslash 2^{k} B\right)} & \leq \frac{C(N)}{\left(1+\frac{2^{k} r}{\rho\left(x_{0}\right)}\right)^{N}}\left(\frac{r^{c_{0}} 2^{k} r\left|2^{k} B\right|^{1 / q-1}}{\left(2^{k} r\right)^{d+c_{0}-1}} \frac{1}{\left(1+\frac{2^{k+2} r}{\rho\left(x_{0}\right)}\right)^{N_{0}}}\right. \\
& \left.\int_{B\left(x_{0}, 2^{k+2} r\right)} V(z) d z+\frac{2^{-k c_{0}}}{\left|2^{k} B\right|}\left|2^{k+1} B\right|^{1 / q}\right) \\
& \leq \frac{C(N)}{\left(1+2^{k} r / \rho\left(x_{0}\right)\right)^{N}} 2^{-k c_{0}}\left|2^{k} B\right|^{1 / q-1},
\end{aligned}
$$

where $N_{0}=\log _{2} C_{0}+1$ with $C_{0}$ the constant in (2.1). This completes the proof. 
Proof of Lemma 5.13. Note that $r \leq \mathcal{C}_{L} \rho\left(x_{0}\right)$ since $a$ is a $\left(H_{L}^{1}, q\right)$-atom related to the ball $B=B\left(x_{0}, r\right)$; and $a$ is $\mathcal{C}_{L}{ }^{c_{0}}$ times a generalized $\left(H_{L}^{1}, q, c_{0}\right)$-atom related to the ball $B=B\left(x_{0}, r\right)$ (see Remark 2.4). In (5.7), we choose $N=\left(k_{0}+1\right) \theta$. Then, Hölder's inequality and Lemma 6.6 give

$$
\begin{aligned}
& \left\|\left(g-g_{B}\right) R_{j}(a)\right\|_{L^{1}}=\left\|\left(g-g_{B}\right) R_{j}(a)\right\|_{L^{1}\left(2^{4} B\right)}+\sum_{k=4}^{\infty}\left\|\left(g-g_{B}\right) R_{j}(a)\right\|_{L^{1}\left(2^{k+1} B \backslash 2^{k} B\right)} \\
& \leq\left\|g-g_{B}\right\|_{\left.L^{q^{\prime}\left(2^{4} B\right.}\right)}\left\|R_{j}\right\|_{L^{q} \rightarrow L^{q}}\|a\|_{L^{q}} \\
& \quad+\sum_{k=4}^{\infty}\left\|g-g_{B}\right\|_{L^{q^{\prime}\left(2^{k+1} B \backslash 2^{k} B\right)}}\left\|R_{j}(a)\right\|_{L^{q}\left(2^{k+1} B \backslash 2^{k} B\right)} \\
& \leq C\|g\|_{\mathrm{BMO}_{L, \theta}}+C \sum_{k=4}^{\infty}(k+1)\left|2^{k+1} B\right|^{1 / q^{\prime}}\left(1+\frac{2^{k+1} r}{\rho(x)}\right)^{\left(k_{0}+1\right) \theta} \\
& \quad \cdot\|g\|_{\mathrm{BMO}_{L, \theta}} \frac{1}{\left(1+\frac{2^{k} r}{\rho(x)}\right)^{\left(k_{0}+1\right) \theta}} 2^{-k c_{0}}\left|2^{k} B\right|^{1 / q-1} \\
& \leq C\|g\|_{\mathrm{BMO}_{L, \theta},}
\end{aligned}
$$

where $1 / q+1 / q^{\prime}=1$. Similarly, we also obtain that

$$
\begin{aligned}
&\left\|\left(f-f_{B}\right)\left(g-g_{B}\right) R_{j}(a)\right\|_{L^{1}}=\left\|\left(f-f_{B}\right)\left(g-g_{B}\right) R_{j}(a)\right\|_{L^{1}\left(2^{4} B\right)} \\
& \quad+\sum_{k=4}^{\infty}\left\|\left(f-f_{B}\right)\left(g-g_{B}\right) R_{j}(a)\right\|_{L^{1}\left(2^{k+1} B \backslash 2^{k} B\right)} \\
& \leq\left\|f-f_{B}\right\|_{L^{2 q^{\prime}\left(2^{4} B\right)}}\left\|g-g_{B}\right\|_{L^{2 q^{\prime}\left(2^{4} B\right)}}\left\|R_{j}(a)\right\|_{L^{q}} \\
&+\sum_{k=4}^{\infty}\left\|f-f_{B}\right\|_{L^{2 q^{\prime}\left(2^{k+1} B\right)}}\left\|g-g_{B}\right\|_{L^{2 q^{\prime}\left(2^{k+1} B\right)}}\left\|R_{j}(a)\right\|_{L^{q}\left(2^{k+1} B \backslash 2^{k} B\right)} \\
& \leq C\|f\|_{\mathrm{BMO}}\|g\|_{\mathrm{BMO}_{L, \theta},},
\end{aligned}
$$

which ends the proof.

\section{Some applications}

The purpose of this section is to give some applications of the decomposition theorems (Theorem 3.1 and Theorem 3.4). To be more precise, we give some subspaces of $H_{L}^{1}\left(\mathbb{R}^{d}\right)$, which do not necessarily depend on $b$ and $T$, such that all commutators $[b, T]$, for $b \in \mathrm{BMO}\left(\mathbb{R}^{d}\right)$ and $T \in \mathcal{K}_{L}$, map continuously these spaces into $L^{1}\left(\mathbb{R}^{d}\right)$.

Especially, using Theorem 3.1 and Theorem 3.4, we find the largest subspace $\mathcal{H}_{L, b}^{1}\left(\mathbb{R}^{d}\right)$ of $H_{L}^{1}\left(\mathbb{R}^{d}\right)$ so that all commutators of Schrödinger-Calderón-Zygmund operators and the Riesz transforms are bounded from $\mathcal{H}_{L, b}^{1}\left(\mathbb{R}^{d}\right)$ into $L^{1}\left(\mathbb{R}^{d}\right)$. Also, it allows to find all functions $b$ in $\operatorname{BMO}\left(\mathbb{R}^{d}\right)$ so that $\mathcal{H}_{L, b}^{1}\left(\mathbb{R}^{d}\right) \equiv H_{L}^{1}\left(\mathbb{R}^{d}\right)$. 


\subsection{Atomic Hardy spaces related to $b \in \operatorname{BMO}\left(\mathbb{R}^{d}\right)$}

Definition 7.1. Let $1<q \leq \infty, \varepsilon>0$ and $b \in \operatorname{BMO}\left(\mathbb{R}^{d}\right)$. A function $a$ is called a $\left(H_{L, b}^{1}, q, \varepsilon\right)$-atom related to the ball $B=B\left(x_{0}, r\right)$ if $a$ is a generalized $\left(H_{L}^{1}, q, \varepsilon\right)$-atom related to the same ball $B$ and

$$
\left|\int_{\mathbb{R}^{d}} a(x)\left(b(x)-b_{B}\right) d x\right| \leq\left(\frac{r}{\rho\left(x_{0}\right)}\right)^{\varepsilon} .
$$

As usual, the space $H_{L, b}^{1, q, \varepsilon}\left(\mathbb{R}^{d}\right)$ is defined as $\mathbb{H}_{L, \text { at }}^{1, q, \varepsilon}\left(\mathbb{R}^{d}\right)$ with generalized $\left(H_{L}^{1}, q, \varepsilon\right)$ atoms replaced by $\left(H_{L, b}^{1}, q, \varepsilon\right)$-atoms.

Obviously, $H_{L, b}^{1, q, \varepsilon}\left(\mathbb{R}^{d}\right) \subset \mathbb{H}_{L, \text { at }}^{1, q, \varepsilon}\left(\mathbb{R}^{d}\right) \equiv H_{L}^{1}\left(\mathbb{R}^{d}\right)$ and the inclusion is continuous.

Theorem 7.2. Let $1<q \leq \infty, \varepsilon>0, b \in \operatorname{BMO}\left(\mathbb{R}^{d}\right)$ and $T \in \mathcal{K}_{L}$. Then, the commutator $[b, T]$ is bounded from $H_{L, b}^{1, q, \varepsilon}\left(\mathbb{R}^{d}\right)$ into $L^{1}\left(\mathbb{R}^{d}\right)$.

Remark 7.3. The space $H_{b}^{1}\left(\mathbb{R}^{d}\right)$ which has been considered by Tang and Bi [44] is a strict subspace of $H_{L, b}^{1, q, \varepsilon}\left(\mathbb{R}^{d}\right)$ in general. As an example, let us take $1<q \leq \infty$, $\varepsilon>0, L=-\Delta+1$, and $b$ be a non-constant bounded function, then it is easy to check that the function $f=\chi_{B(0,1)}$ belongs to $H_{L, b}^{1, q, \varepsilon}\left(\mathbb{R}^{d}\right)$ but not to $H_{b}^{1}\left(\mathbb{R}^{d}\right)$. Thus, Theorem 7.2 can be seen as an improvement of the main result of [44].

We should also point out that the authors in [44] proved their main result (see [44], Theorem 3.1) by establishing that

$$
\left\|\left[b, R_{j}\right](a)\right\|_{L^{1}} \leq C\|b\|_{\mathrm{BMO}}
$$

for all $H_{b}^{1}$-atom $a$. However, as pointed in [8] and [28], such arguments are not sufficient to conclude that $\left[b, R_{j}\right]$ is bounded from $H_{b}^{1}\left(\mathbb{R}^{d}\right)$ into $L^{1}\left(\mathbb{R}^{d}\right)$ in general.

Proof of Theorem 7.2. Let $a$ be a $\left(H_{L, b}^{1}, q, \varepsilon\right)$-atom related to the ball $B=B\left(x_{0}, r\right)$. We first prove that $\left(b-b_{B}\right) a$ is $C\|b\|_{\text {BMO }}$ times a generalized $\left(H_{L}^{1},(\widetilde{q}+1) / 2, \varepsilon\right)$ atom, where $\widetilde{q} \in(1, \infty)$ will be defined later and the positive constant $C$ is independent of $b, a$. Indeed, one has supp $\left(b-b_{B}\right) a \subset \operatorname{supp} a \subset B$. In addition, from Hölder inequality and John-Nirenberg (classical) inequality,

$$
\left\|\left(b-b_{B}\right) a\right\|_{L^{(\widetilde{q}+1) / 2}} \leq\left\|\left(b-b_{B}\right) \chi_{B}\right\|_{L^{\widetilde{q}(\widetilde{q}+1) /(\widetilde{q}-1)}}\|a\|_{L^{\widetilde{q}}} \leq C\|b\|_{\mathrm{BMO}}|B|^{(-\widetilde{q}+1) /(\widetilde{q}+1)},
$$

where $\widetilde{q}=q$ if $1<q<\infty$ and $\widetilde{q}=2$ if $q=\infty$. These together with (7.1) yield that $\left(b-b_{B}\right) a$ is $C\|b\|_{\text {BMO }}$ times a generalized $\left(H_{L}^{1},(\widetilde{q}+1) / 2, \varepsilon\right)$-atom, and thus $\left\|\left(b-b_{B}\right) a\right\|_{H_{L}^{1}} \leq C\|b\|_{\mathrm{BMO}}$.

We now prove that $\mathfrak{S}(a, b)$ belongs to $H_{L}^{1}\left(\mathbb{R}^{d}\right)$.

By Theorem 3.4, there exist, for $j=1, \ldots, d$, bounded bilinear operators $\mathfrak{R}_{j}: H_{L}^{1}\left(\mathbb{R}^{d}\right) \times \operatorname{BMO}\left(\mathbb{R}^{d}\right) \rightarrow L^{1}\left(\mathbb{R}^{d}\right)$, such that

$$
\left[b, R_{j}\right](a)=\mathfrak{R}_{j}(a, b)+R_{j}(\mathfrak{S}(a, b)),
$$


since $R_{j}$ is linear and belongs to $\mathcal{K}_{L}$ (see Proposition 4.2). Consequently, for every $j=1, \ldots, d$, as $R_{j} \in \mathcal{K}_{L}$,

$$
\begin{aligned}
\left\|R_{j}(\mathfrak{S}(a, b))\right\|_{L^{1}} & =\left\|\left(b-b_{B}\right) R_{j}(a)-R_{j}\left(\left(b-b_{B}\right) a\right)-\mathfrak{R}_{j}(a, b)\right\|_{L^{1}} \\
& \leq\left\|\left(b-b_{B}\right) R_{j}(a)\right\|_{L^{1}}+\left\|R_{j}\right\|_{H_{L}^{1} \rightarrow L^{1}}\left\|\left(b-b_{B}\right) a\right\|_{H_{L}^{1}}+\left\|\Re_{j}(a, b)\right\|_{L^{1}} \\
& \leq C\|b\|_{\mathrm{BMO}} .
\end{aligned}
$$

This together with Proposition 5.6 prove that $\mathfrak{S}(a, b) \in H_{L}^{1}\left(\mathbb{R}^{d}\right)$, and moreover that

$$
\|\mathfrak{S}(a, b)\|_{H_{L}^{1}} \leq C\|b\|_{\mathrm{BMO}}
$$

Now, for any $f \in H_{L, b}^{1, q, \varepsilon}\left(\mathbb{R}^{d}\right)$, there exists an expansion $f=\sum_{k=1}^{\infty} \lambda_{k} a_{k}$ where the $a_{k}$ are $\left(H_{L, b}^{1}, q, \varepsilon\right)$-atoms and $\sum_{k=1}^{\infty}\left|\lambda_{k}\right| \leq 2\|f\|_{H_{L, b}^{1, q, \varepsilon}}$. Then, the sequence $\left\{\sum_{k=1}^{n} \lambda_{k} a_{k}\right\}_{n \geq 1}$ converges to $f$ in $H_{L, b}^{1, q, \varepsilon}\left(\mathbb{R}^{d}\right)$ and thus in $H_{L}^{1}\left(\mathbb{R}^{d}\right)$. Hence, Proposition 5.6 implies that the sequence $\left\{\mathfrak{S}\left(\sum_{k=1}^{n} \lambda_{k} a_{k}, b\right)\right\}_{n \geq 1}$ converges to $\mathfrak{S}(f, b)$ in $L^{1}\left(\mathbb{R}^{d}\right)$. In addition, by $(7.2)$,

$$
\left\|\mathfrak{S}\left(\sum_{k=1}^{n} \lambda_{k} a_{k}, b\right)\right\|_{H_{L}^{1}} \leq \sum_{k=1}^{n}\left|\lambda_{k}\right|\left\|\mathfrak{S}\left(a_{k}, b\right)\right\|_{H_{L}^{1}} \leq C\|f\|_{H_{L, b}^{1, q, \varepsilon}}\|b\|_{\mathrm{BMO}} .
$$

We then use Theorem 3.1 and the weak-star convergence in $H_{L}^{1}\left(\mathbb{R}^{d}\right)$ (see [29]) to conclude that

$$
\begin{aligned}
\|[b, T](f)\|_{L^{1}} & \leq\left\|\Re_{T}(f, b)\right\|_{L^{1}}+\|T\|_{H_{L}^{1} \rightarrow L^{1}}\|\mathfrak{S}(f, b)\|_{H_{L}^{1}} \\
& \leq C\|f\|_{H_{L}^{1}}\|b\|_{\mathrm{BMO}}+C\|f\|_{H_{L, b}^{1, q, \varepsilon}}\|b\|_{\mathrm{BMO}} \leq C\|f\|_{H_{L, b}^{1, q, \varepsilon}}\|b\|_{\mathrm{BMO}},
\end{aligned}
$$

which ends the proof.

\subsection{The spaces $\mathcal{H}_{L, b}^{1}\left(\mathbb{R}^{d}\right)$ related to $b \in \mathrm{BMO}\left(\mathbb{R}^{d}\right)$}

In this section, we find the largest subspace $\mathcal{H}_{L, b}^{1}\left(\mathbb{R}^{d}\right)$ of $H_{L}^{1}\left(\mathbb{R}^{d}\right)$ so that all commutators of Schrödinger-Calderón-Zygmund operators and the Riesz transforms are bounded from $\mathcal{H}_{L, b}^{1}\left(\mathbb{R}^{d}\right)$ into $L^{1}\left(\mathbb{R}^{d}\right)$. Also, we find all functions $b$ in $\operatorname{BMO}\left(\mathbb{R}^{d}\right)$ so that $\mathcal{H}_{L, b}^{1}\left(\mathbb{R}^{d}\right) \equiv H_{L}^{1}\left(\mathbb{R}^{d}\right)$.

Definition 7.4. Let $b$ be a non-constant BMO-function. The space $\mathcal{H}_{L, b}^{1}\left(\mathbb{R}^{d}\right)$ consists of all $f$ in $H_{L}^{1}\left(\mathbb{R}^{d}\right)$ such that $\left[b, \mathcal{M}_{L}\right](f)(x)=\mathcal{M}_{L}(b(x) f(\cdot)-b(\cdot) f(\cdot))(x)$ belongs to $L^{1}\left(\mathbb{R}^{d}\right)$. We equipped $\mathcal{H}_{L, b}^{1}\left(\mathbb{R}^{d}\right)$ with the norm

$$
\|f\|_{\mathcal{H}_{L, b}^{1}}=\|f\|_{H_{L}^{1}}\|b\|_{\mathrm{BMO}}+\left\|\left[b, \mathcal{M}_{L}\right](f)\right\|_{L^{1}} .
$$

Here, we just consider non-constant functions $b$ in $\operatorname{BMO}\left(\mathbb{R}^{d}\right)$ since $[b, T]=0$ if $b$ is a constant function. 
Theorem 7.5. Let $b$ be a non-constant BMO-function. Then, the following statements hold:

(i) For every $T \in \mathcal{K}_{L}$, the commutator $[b, T]$ is bounded from $\mathcal{H}_{L, b}^{1}\left(\mathbb{R}^{d}\right)$ into $L^{1}\left(\mathbb{R}^{d}\right)$.

(ii) Assume that $\mathcal{X}$ is a subspace of $H_{L}^{1}\left(\mathbb{R}^{d}\right)$ such that all commutators of the Riesz transforms are bounded from $\mathcal{X}$ into $L^{1}\left(\mathbb{R}^{d}\right)$. Then, $\mathcal{X} \subset \mathcal{H}_{L, b}^{1}\left(\mathbb{R}^{d}\right)$.

(iii) $\mathcal{H}_{L, b}^{1}\left(\mathbb{R}^{d}\right) \equiv H_{L}^{1}\left(\mathbb{R}^{d}\right)$ if and only if $b \in \mathrm{BMO}_{L}^{\log }\left(\mathbb{R}^{d}\right)$.

To prove Theorem 7.5, we need the following lemma.

Lemma 7.6. Let $b$ be a non-constant BMO-function and $f \in H_{L}^{1}\left(\mathbb{R}^{d}\right)$. Then, the following conditions are equivalent:

(i) $f \in \mathcal{H}_{L, b}^{1}\left(\mathbb{R}^{d}\right)$.

(ii) $\mathfrak{S}(f, b) \in H_{L}^{1}\left(\mathbb{R}^{d}\right)$.

(iii) $\left[b, R_{j}\right](f) \in L^{1}\left(\mathbb{R}^{d}\right)$ for all $j=1, \ldots, d$.

Furthermore, if one of these conditions is satisfied, then

$$
\begin{aligned}
\|f\|_{\mathcal{H}_{L, b}^{1}}=\|f\|_{H_{L}^{1}}\|b\|_{\mathrm{BMO}}+\left\|\left[b, \mathcal{M}_{L}\right](f)\right\|_{L^{1}} & \approx\|f\|_{H_{L}^{1}}\|b\|_{\mathrm{BMO}}+\|\mathfrak{S}(f, b)\|_{H_{L}^{1}} \\
& \approx\|f\|_{H_{L}^{1}}\|b\|_{\mathrm{BMO}}+\sum_{j=1}^{d}\left\|\left[b, R_{j}\right](f)\right\|_{L^{1}},
\end{aligned}
$$

where the constants are independent of $b$ and $f$.

Proof. (i) $\Leftrightarrow$ (ii). As $\mathcal{M}_{L} \in \mathcal{K}_{L}$ (see Proposition 4.5), by Theorem 3.1, there is a bounded subbilinear operator $\mathfrak{R}: H_{L}^{1}\left(\mathbb{R}^{d}\right) \times \operatorname{BMO}\left(\mathbb{R}^{d}\right) \rightarrow L^{1}\left(\mathbb{R}^{d}\right)$ such that

$$
\mathcal{M}_{L}(\mathfrak{S}(f, b))-\mathfrak{R}(f, b) \leq\left|\left[b, \mathcal{M}_{L}\right](f)\right| \leq \mathcal{M}_{L}(\mathfrak{S}(f, b))+\mathfrak{R}(f, b) .
$$

Consequently, $\left[b, \mathcal{M}_{L}\right](f) \in L^{1}\left(\mathbb{R}^{d}\right)$ if and only if $\mathfrak{S}(f, b) \in H_{L}^{1}\left(\mathbb{R}^{d}\right)$; moreover,

$$
\|f\|_{\mathcal{H}_{L, b}^{1}} \approx\|f\|_{H_{L}^{1}}\|b\|_{\mathrm{BMO}}+\|\mathfrak{S}(f, b)\|_{H_{L}^{1}} .
$$

(ii) $\Leftrightarrow$ (iii). As the Riesz transforms $R_{j}$ are in $\mathcal{K}_{L}$ (see Proposition 4.2), by Theorem 3.4, there are $d$ bounded subbilinear operator $\Re_{j}: H_{L}^{1}\left(\mathbb{R}^{d}\right) \times \operatorname{BMO}\left(\mathbb{R}^{d}\right) \rightarrow$ $L^{1}\left(\mathbb{R}^{d}\right), j=1, \ldots, d$, such that

$$
\left[b, R_{j}\right](f)=\mathfrak{R}_{j}(f, b)+R_{j}(\mathfrak{S}(f, b)) .
$$

Therefore, $\mathfrak{S}(f, b) \in H_{L}^{1}\left(\mathbb{R}^{d}\right)$ if and only if $\left[b, R_{j}\right](f) \in L^{1}\left(\mathbb{R}^{d}\right)$ for all $j=1, \ldots, d$; moreover,

$$
\|f\|_{H_{L}^{1}}\|b\|_{\mathrm{BMO}}+\|\mathfrak{S}(f, b)\|_{H_{L}^{1}} \approx\|f\|_{H_{L}^{1}}\|b\|_{\mathrm{BMO}}+\sum_{j=1}^{d}\left\|\left[b, R_{j}\right](f)\right\|_{L^{1}} .
$$


Proof of Theorem 7.5. By Theorem 3.1, there is a bounded subbilinear operator $\mathfrak{R}_{T}: H_{L}^{1}\left(\mathbb{R}^{d}\right) \times \operatorname{BMO}\left(\mathbb{R}^{d}\right) \rightarrow L^{1}\left(\mathbb{R}^{d}\right)$ such that

$$
|T(\mathfrak{S}(f, b))|-\mathfrak{R}_{T}(f, b) \leq|[b, T](f)| \leq|T(\mathfrak{S}(f, b))|+\mathfrak{R}_{T}(f, b) .
$$

Applying Lemma 7.6 gives for every $f \in \mathcal{H}_{L, b}^{1}\left(\mathbb{R}^{d}\right)$,

$$
\begin{aligned}
\|[b, T](f)\|_{L^{1}} & \leq\|T\|_{H_{L}^{1} \rightarrow L^{1}}\|\mathfrak{S}(f, b)\|_{H_{L}^{1}}+\left\|\Re_{T}(f, b)\right\|_{L^{1}} \\
& \leq C\|f\|_{\mathcal{H}_{L, b}^{1}}+C\|f\|_{H_{L}^{1}}\|b\|_{\mathrm{BMO}} \leq C\|f\|_{\mathcal{H}_{L, b}^{1}} .
\end{aligned}
$$

Therefore, $[b, T]$ is bounded from $\mathcal{H}_{L, b}^{1}\left(\mathbb{R}^{d}\right)$ into $L^{1}\left(\mathbb{R}^{d}\right)$. This ends the proof of (i).

The proof of (ii) follows directly from Lemma 7.6.

The proof of (iii) follows directly from Theorem 3.6 and Lemma 7.6.

\subsection{Atomic Hardy spaces $H_{L, \alpha}^{\log }\left(\mathbb{R}^{d}\right)$}

Definition 7.7. Let $\alpha \in \mathbb{R}$. We say that the function $a$ is a $H_{L, \alpha}^{\log }$-atom related to the ball $B=B\left(x_{0}, r\right)$ if

(i) supp $a \subset B$,

(ii) $\|a\|_{L^{2}} \leq\left(\log \left(e+\frac{\rho\left(x_{0}\right)}{r}\right)\right)^{\alpha}|B|^{-1 / 2}$,

(iii) $\int_{\mathbb{R}^{d}} a(x) d x=0$.

As usual, the space $H_{L, \alpha}^{\log }\left(\mathbb{R}^{d}\right)$ is defined as $\mathbb{H}_{L, \text { at }}^{1, q, \varepsilon}$ with generalized $\left(H_{L}^{1}, q, \varepsilon\right)$ atoms replaced by $H_{L, \alpha}^{\mathrm{log}}$-atoms.

Clearly, $H_{L, 0}^{\log }\left(\mathbb{R}^{d}\right)$ is just $H^{1}\left(\mathbb{R}^{d}\right) \subset H_{L}^{1}\left(\mathbb{R}^{d}\right)$. Moreover, $H_{L, \alpha}^{\log }\left(\mathbb{R}^{d}\right) \subset H_{L, \alpha^{\prime}}^{\log }\left(\mathbb{R}^{d}\right)$ for all $\alpha \leq \alpha^{\prime}$. It should be pointed out that when $L=-\Delta+1$ and $\alpha \geq 0$, then $H_{L, \alpha}^{\log }\left(\mathbb{R}^{d}\right)$ is just the space of all distributions $f$ such that

$$
\int_{\mathbb{R}^{d}} \frac{\mathfrak{M} f(x) / \lambda}{(\log (e+\mathfrak{M} f(x) / \lambda))^{\alpha}} d x<\infty
$$

for some $\lambda>0$. Moreover (see [27] for the details),

$$
\|f\|_{H_{L, \alpha}^{\log }} \approx \inf \left\{\lambda>0: \int_{\mathbb{R}^{d}} \frac{\mathfrak{M} f(x) / \lambda}{(\log (e+\mathfrak{M} f(x) / \lambda))^{\alpha}} d x \leq 1\right\} .
$$

Theorem 7.8. For every $T \in \mathcal{K}_{L}$ and $b \in \mathrm{BMO}\left(\mathbb{R}^{d}\right)$, the commutator $[b, T]$ is bounded from $H_{L,-1}^{\log }\left(\mathbb{R}^{d}\right)$ into $L^{1}\left(\mathbb{R}^{d}\right)$.

Proof. Let $a$ be a $H_{L,-1}^{\text {log }}$-atom related to the ball $B=B\left(x_{0}, r\right)$. Let us first prove that $\left(b-b_{B}\right) a \in H_{L}^{1}\left(\mathbb{R}^{d}\right)$. As $H_{L}^{1}\left(\mathbb{R}^{d}\right)$ is the dual of $\mathrm{VMO}_{L}\left(\mathbb{R}^{d}\right)$ (see Theorem 5.8), it is sufficient to show that for every $g \in C_{c}^{\infty}\left(\mathbb{R}^{d}\right)$,

$$
\left\|\left(b-b_{B}\right) a g\right\|_{L^{1}} \leq C\|b\|_{\mathrm{BMO}}\|g\|_{\mathrm{BMO}_{L}} .
$$


Indeed, using the estimate $\left|g_{B}\right| \leq C \log \left(e+\rho\left(x_{0}\right) / r\right)\|g\|_{\mathrm{BMO}_{L}}$ (see Lemma 2 of [15]), the Hölder inequality and the classical John-Nirenberg inequality give

$$
\begin{aligned}
\left\|\left(b-b_{B}\right) a g\right\|_{L^{1}} \leq & \left\|\left(g-g_{B}\right)\left(b-b_{B}\right) a\right\|_{L^{1}}+\left|g_{B}\right|\left\|\left(b-b_{B}\right) a\right\|_{L^{1}} \\
\leq & \left\|\left(g-g_{B}\right) \chi_{B}\right\|_{L^{4}}\left\|\left(b-b_{B}\right) \chi_{B}\right\|_{L^{4}}\|a\|_{L^{2}} \\
& +C \log \left(e+\frac{\rho\left(x_{0}\right)}{r}\right)\|g\|_{\mathrm{BMO}_{L}}\left\|\left(b-b_{B}\right) \chi_{B}\right\|_{L^{2}}\|a\|_{L^{2}} \\
\leq & C\|b\|_{\mathrm{BMO}}\|g\|_{\mathrm{BMO}_{L}},
\end{aligned}
$$

which proves that $\left(b-b_{B}\right) a \in H_{L}^{1}\left(\mathbb{R}^{d}\right)$, moreover, $\left\|\left(b-b_{B}\right) a\right\|_{H_{L}^{1}} \leq C\|b\|_{\text {BMO }}$.

Similarly to the proof of Theorem 7.2, we also obtain that

$$
\|\mathfrak{S}(f, b)\|_{H_{L}^{1}} \leq C\|f\|_{H_{L,-1}^{\log }}\|b\|_{\text {BMO }}
$$

for all $f \in H_{L,-1}^{\log }\left(\mathbb{R}^{d}\right)$. Therefore, Theorem 3.1 allows to conclude that

$$
\|[b, T](f)\|_{L^{1}} \leq C\|f\|_{H_{L,-1}^{\log }}\|b\|_{\mathrm{BMO}}
$$

which ends the proof.

As a consequence of the proof of Theorem 7.8, we obtain the following result.

Proposition 7.9. Let $T \in \mathcal{K}_{L}$. Then, $\mathfrak{T}(f, b):=[b, T](f)$ is a bounded subbilinear operator from $H_{L,-1}^{\log }\left(\mathbb{R}^{d}\right) \times \mathrm{BMO}\left(\mathbb{R}^{d}\right)$ into $L^{1}\left(\mathbb{R}^{d}\right)$.

\subsection{The Hardy-Sobolev space $H_{L}^{1,1}\left(\mathbb{R}^{d}\right)$}

Following Hofmann et al. [23], we say that $f$ belongs to the (inhomogeneous) Hardy-Sobolev $H_{L}^{1,1}\left(\mathbb{R}^{d}\right)$ if $f, \partial_{x_{1}} f, \ldots, \partial_{x_{d}} f \in H_{L}^{1}\left(\mathbb{R}^{d}\right)$. Then, the norm on $H_{L}^{1,1}\left(\mathbb{R}^{d}\right)$ is defined by

$$
\|f\|_{H_{L}^{1,1}}=\|f\|_{H_{L}^{1}}+\sum_{j=1}^{d}\left\|\partial_{x_{j}} f\right\|_{H_{L}^{1}} .
$$

It should be pointed out that the authors in [23] proved that the space $H_{-\Delta}^{1,1}\left(\mathbb{R}^{d}\right)$ is just the classical (inhomogeneous) Hardy-Sobolev $H^{1,1}\left(\mathbb{R}^{d}\right.$ ) (see for example [1]), and can be identified with the (inhomogeneous) Triebel-Lizorkin space $F_{1}^{1,2}\left(\mathbb{R}^{d}\right)$ (see [26]). More precisely, $f$ belongs to $H^{1,1}\left(\mathbb{R}^{d}\right)$ if and only if

$$
\mathcal{W}_{\psi}(f)=\left\{\sum_{I} \sum_{\sigma \in \mathcal{E}}\left|\left\langle f, \psi_{I}^{\sigma}\right\rangle\right|^{2}\left(1+|I|^{-1 / d}\right)^{2}|I|^{-1} \chi_{I}\right\}^{1 / 2} \in L^{1}\left(\mathbb{R}^{d}\right),
$$

moreover,

$$
\|f\|_{H^{1,1}} \approx\left\|\mathcal{W}_{\psi}(f)\right\|_{L^{1}}
$$

Here $\left\{\psi^{\sigma}\right\}_{\sigma \in \mathcal{E}}$ is the wavelet as in Section 4 . 
Theorem 7.10. Let $L=-\Delta+1$. Then, for every $T \in \mathcal{K}_{L}$ and $b \in \mathrm{BMO}\left(\mathbb{R}^{d}\right)$, the commutator $[b, T]$ is bounded from $H_{L}^{1,1}\left(\mathbb{R}^{d}\right)$ into $L^{1}\left(\mathbb{R}^{d}\right)$.

Remark 7.11. When $L=-\Delta+1$, we can define $\mathfrak{H}(f)=f-\varphi * f$ instead of $\mathfrak{H}(f)=\sum_{n, k}\left(\psi_{n, k} f-\varphi_{2^{-n / 2}} *\left(\psi_{n, k} f\right)\right)$ as in Section 5. In other words, the bilinear operator $\mathfrak{S}$ in Theorem 3.1 and Theorem 3.4 can be defined as $\mathfrak{S}(f, g)=$ $-\Pi(f-\varphi * f, g)$. As $\mathfrak{H}(f)=f-\varphi * f$, it is easy to see that

$$
\partial_{x_{j}}(\mathfrak{H}(f))=\mathfrak{H}\left(\partial_{x_{j}} f\right) \text {. }
$$

Here and in what follows, for any dyadic cube $Q=Q(y, r):=\left\{x \in \mathbb{R}^{d}:-r \leq\right.$ $x_{j}-y_{j}<r$ for all $\left.j=1, \ldots, d\right\}$, we denote by $B_{Q}$ the ball

$$
B_{Q}:=\left\{x \in \mathbb{R}^{d}:|x-y|<2 \sqrt{d} r\right\} .
$$

To prove Theorem 7.10, we need the following lemma.

Lemma 7.12. Let $L=-\Delta+1$. Then, the bilinear operator $\Pi$ maps continuously $H^{1,1}\left(\mathbb{R}^{d}\right) \times \operatorname{BMO}\left(\mathbb{R}^{d}\right)$ into $H_{L}^{1}\left(\mathbb{R}^{d}\right)$.

Proof. Note that $\rho(x)=1$ for all $x \in \mathbb{R}^{d}$ since $V(x) \equiv 1$. We first claim that there exists a constant $C>0$ such that

$$
\left\|\left(1+|I|^{-1 / d}\right)^{-1}\left(\psi_{I}^{\sigma}\right)^{2}\right\|_{H_{L}^{1}} \leq C
$$

for all dyadic $I=Q\left[x_{0}, r\right)$ and $\sigma \in \mathcal{E}$. Indeed, it follows from Remark 5.2 that supp $\left(1+|I|^{-1 / d}\right)^{-1}\left(\psi_{I}^{\sigma}\right)^{2} \subset c I \subset c B_{I}$, and it is clear that $\left\|\left(1+|I|^{-1 / d}\right)^{-1}\left(\psi_{I}^{\sigma}\right)^{2}\right\|_{L^{\infty}} \leq$ $|I|^{-1}\|\psi\|_{L^{\infty}} \leq C\left|c B_{I}\right|^{-1}$. In addition,

$$
\left|\int_{\mathbb{R}^{d}}\left(1+|I|^{-1 / d}\right)^{-1}\left(\psi_{I}^{\sigma}(x)\right)^{2} d x\right|=\left(1+|I|^{-1 / d}\right)^{-1} \leq C \frac{r}{\rho\left(x_{0}\right)} .
$$

Hence, $\left(1+|I|^{-1 / d}\right)^{-1}\left(\psi_{I}^{\sigma}\right)^{2}$ is $C$ times a generalized $\left(H_{L}^{1}, \infty, 1\right)$-atom related to the ball $c B_{I}$, and thus (7.4) holds.

Now, for every $(f, g) \in H^{1,1}\left(\mathbb{R}^{d}\right) \times \operatorname{BMO}\left(\mathbb{R}^{d}\right)$, (7.4) implies that

$$
\begin{aligned}
\|\Pi(f, g)\|_{H_{L}^{1}} & =\left\|\sum_{I} \sum_{\sigma \in \mathcal{E}}\left\langle f, \psi_{I}^{\sigma}\right\rangle\left\langle g, \psi_{I}^{\sigma}\right\rangle\left(\psi_{I}^{\sigma}\right)^{2}\right\|_{H_{L}^{1}} \\
& \leq C \sum_{I} \sum_{\sigma \in \mathcal{E}}\left(\left|\left\langle f, \psi_{I}^{\sigma}\right\rangle\right|\left(1+|I|^{-1 / d}\right)\right)\left|\left\langle g, \psi_{I}^{\sigma}\right\rangle\right| \\
& \leq C\left\|\mathcal{W}_{\psi}(f)\right\|_{L^{1}}\|g\|_{\dot{F}_{\infty}^{0,2}} \leq C\|f\|_{H^{1,1}}\|g\|_{\mathrm{BMO}}
\end{aligned}
$$

where we have used the fact that $\operatorname{BMO}\left(\mathbb{R}^{d}\right) \equiv \dot{F}_{\infty}^{0,2}\left(\mathbb{R}^{d}\right)$ is the dual of $H^{1}\left(\mathbb{R}^{d}\right) \equiv$ $\dot{F}_{1}^{0,2}\left(\mathbb{R}^{d}\right)$, we refer the reader to [18] for more details.

Proof of Theorem 7.10. Let $(f, b) \in H_{L}^{1,1}\left(\mathbb{R}^{d}\right) \times \mathrm{BMO}\left(\mathbb{R}^{d}\right)$. Thanks to Lemma 7.12, Remark 7.11 and Lemma 5.4, we get

$$
\|\mathfrak{S}(f, b)\|_{H_{L}^{1}} \leq C\|\mathfrak{H}(f)\|_{H^{1,1}}\|b\|_{\mathrm{BMO}} \leq C\|f\|_{H_{L}^{1,1}}\|b\|_{\mathrm{BMO}}
$$


Then we use Theorem 3.1 to conclude that

$$
\|[b, T](f)\|_{L^{1}} \leq\left\|\mathfrak{R}_{T}(f, b)\right\|_{L^{1}}+\|T\|_{H_{L}^{1} \rightarrow L^{1}}\|\mathfrak{S}(f, b)\|_{H_{L}^{1}} \leq C\|f\|_{H_{L}^{1,1}}\|b\|_{\mathrm{BMO}},
$$

which ends the proof.

As a consequence of the proof of Theorem 7.10, we obtain the following result.

Proposition 7.13. Let $L=-\Delta+1$ and $T \in \mathcal{K}_{L}$. Then, $\mathfrak{T}(f, b):=[b, T](f)$ is a bounded subbilinear operator from $H_{L}^{1,1}\left(\mathbb{R}^{d}\right) \times \mathrm{BMO}\left(\mathbb{R}^{d}\right)$ into $L^{1}\left(\mathbb{R}^{d}\right)$.

\section{References}

[1] Auscher, P., Russ, E. And Tchamitchian, P.: Hardy Sobolev spaces on strongly Lipschitz domains of $\mathbb{R}^{n}$. J. Funct. Anal. 218 (2005), no. 1, 54-109.

[2] Bernicot, F.: A T(1)-theorem in relation to a semigroup of operators and applications to new paraproducts. Trans. Amer. Math. Soc. 364 (2012), no. 11, 6071-6108.

[3] Betancor, J. J., Crescimbeni, R., Farina, J. C., Stinga, P. R. and Torrea, J. L.: A T1 criterion for Hermite-Calderón-Zygmund operators on the $\mathrm{BMO}_{H}\left(\mathbb{R}^{n}\right)$ space and applications. Ann. Sc. Norm. Super. Pisa Cl. Sci. (5) 12 (2013), no. 1, 157-187.

[4] Bonami, A., Grellier, S. And Ky, L. D.: Paraproducts and products of functions in $\operatorname{BMO}\left(\mathbb{R}^{n}\right)$ and $H^{1}\left(\mathbb{R}^{n}\right)$ through wavelets. J. Math. Pure Appl. (9) 97 (2012), no. 3 , 230-241.

[5] Bonami, A., Grellier, S. And Sehba, B. F.: Boundedness of Hankel operators on $\mathcal{H}^{1}\left(\mathbb{B}^{n}\right)$. C. R. Math. Acad. Sci. Paris 344 (2007), no. 12, 749-752.

[6] Bongionni, B., Harboure, E. and Salinas, O.: Riesz transforms related to Schrödinger operators acting on BMO type spaces. J. Math. Anal. Appl. 357 (2009), no. $1,115-131$.

[7] Bongionni, B., Harboure, E. and Salinas, O.: Commutators of Riesz transforms related to Schrödinger operators. J. Fourier Anal. Appl. 17 (2011), no. 1, $115-134$.

[8] Bownik, M.: Boundedness of operators on Hardy spaces via atomic decompositions. Proc. Amer. Math. Soc. 133 (2005), no. 12, 3535-3542.

[9] Bramanti, M. and Brandolini, L.: Estimates of BMO type for singular integrals on spaces of homogeneous type and applications to hypoelliptic PDEs. Rev. Mat. Iberoamericana 21 (2005), no. 2, 511-556.

[10] Bui, T.A.: Weighted estimates for commutators of some singular integrals related to Schrödinger operators. Bull. Sci. Math. 138 (2014), no. 2, 270-292.

[11] Chang, D-C., Dafni, G. And Stein, E. M.: Hardy spaces, BMO, and boundary value problems for the Laplacian on a smooth domain in $\mathbb{R}^{n}$. Trans. Amer. Math. Soc. 351 (1999), no. 4, 1605-1661.

[12] Coifman, R. R., Rochberg, R. and Weiss, G.: Factorization theorems for Hardy spaces in several variables. Ann. of Math. (2) 103 (1976), no. 3, 611-635.

[13] Dafni, G.: Hardy spaces on strongly pseudoconvex domains in $\mathbb{C}^{n}$ and domains of finite type in $\mathbb{C}^{2}$. Ph. D. Thesis, Princeton University, 1993. 
[14] David, G. And Journé, J-L.: A boundedness criterion for generalized CalderónZygmund operators. Ann. of Math. (2) 120 (1984), no. 2, 371-397.

[15] Dziubański, J., Garrigós, G., Martínez, T., Torrea, J. and Zienkiewicz, J.: BMO spaces related to Schrödinger operators with potentials satisfying a reverse Hölder inequality. Math. Z. 249 (2005), no. 2, 329-356.

[16] Dziubański, J. And Zienkiewicz, J.: Hardy space $H^{1}$ associated to Schrödinger operator with potential satisfying reverse Hölder inequality. Rev. Mat. Iberoamericana 15 (1999), no. 2, 279-296.

[17] Fefferman, C. And Stein, E. M.: $H^{p}$ spaces of several variables. Acta Math. 129 (1972), no. 3-4, 137-193.

[18] Frazier, M. and Jawerth, B.: A discrete transform and decompositions of distribution spaces. J. Funct. Anal. 93 (1990), no. 1, 34-170.

[19] Gehring, F. W.: The $L^{p}$-integrability of the partial derivatives of a quasiconformal mapping. Acta Math. 130 (1973), 265-277.

[20] Goldberg, D.: A local version of real Hardy spaces. Duke J. Math. 46 (1979), no. $1,27-42$.

[21] Guo, Z., Li, P. And Peng, L.: $L^{p}$ boundedness of commutators of Riesz transforms associated to Schrödinger operator. J. Math. Anal. Appl. 341 (2008), no. 1, 421-432.

[22] Hofmann, S., Lu, G., Mitrea, D., Mitrea, M. and Yan, L.: Hardy spaces associated to non-negative self-adjoint operators satisfying Davies-Gaffney estimates. Mem. Amer. Math. Soc. 214 (2011), no. 1007, vi+78.

[23] Hofmann, S., Mayboroda, S. and McIntosh, A.: Second order elliptic operators with complex bounded measurable coeffcients in $L^{p}$, Sobolev and Hardy spaces. Ann. Sci. Éc. Norm. Supér. (4) 44 (2011), no. 5, 723-800.

[24] Janson, S.: On functions with conditions on the mean oscillation. Ark. Mat. 14 (1976), no. 2, 189-196.

[25] Janson, S., Peetre, J. And Semmes, S.: On the action of Hankel and Toeplitz operators on some function spaces. Duke Math. J. 51 (1984), no. 4, 937-958.

[26] Koskela, P., Yang, D. And Zhou, Y.: A characterization of Hajlasz-Sobolev and Triebel-Lizorkin spaces via grand Littlewood-Paley functions. J. Funct. Anal. 258 (2010), no. 8, 2637-2661.

[27] Ky, L. D.: New Hardy spaces of Musielak-Orlicz type and boundedness of sublinear operators. Integral Equations Operator Theory 78 (2014), no. 1, 115-150.

[28] KY, L. D.: Bilinear decompositions and commutators of singular integral operators. Trans. Amer. Math. Soc. 365 (2013), no. 6, 2931-2958.

[29] Ky, L. D.: On weak*-convergence in $H_{L}^{1}\left(\mathbb{R}^{d}\right)$. Potential Anal. 39 (2013), no. 4, $355-368$.

[30] Li, H-Q.: Estimations $L^{p}$ des opérateurs de Schrödinger sur les groupes nilpotents. J. Funct. Anal. 161 (1999), no. 1, 152-218.

[31] Li, P. And Peng, L.: The decomposition of product space $H_{L}^{1} \times \mathrm{BMO}_{L}$. J. Math. Anal. Appl. 349 (2009), no. 2, 484-492.

[32] Li, P. And Peng, L.: Endpoint estimates for commutators of Riesz transforms associated with Schrödinger operators. Bull. Aust. Math. Soc. 82 (2010), no. 3, 367-389. 
[33] Ma, T., Stinga, P. R., Torrea, J. L. and Zhang, C.: Regularity estimates in Hölder spaces for Schrödinger operators via a T1 theorem. Ann. Mat. Pura Appl. (4) 193 (2014), no. 2, 561-589.

[34] Nakai, E.: A generalization of Hardy spaces $H^{p}$ by using atoms. Acta Math. Sin. (Engl. Ser.) 24 (2008), no. 8, 1243-1268.

[35] Paluszyński, M.: Characterization of Lipschitz spaces via the commutator operator of Coifman, Rochberg and Weiss: a multiplier theorem for the semigroup of contractions. Ph.D. Thesis, Washington University in St. Louis, 1992.

[36] Papadimitrakis, M. and Virtanen, J. A.: Hankel and Toeplitz transforms on $H^{1}$ : continuity, compactness and Fredholm properties. Integral Equations Operator Theory 61 (2008), no. 4, 573-591.

[37] PÉrez, C.: Endpoint estimates for commutators of singular integral operators. J. Func. Anal. 128 (1995), no. 1, 163-185.

[38] Pérez, C. and Pradolini, G.: Sharp weighted endpoint estimates for commutators of singular integrals. Michigan Math. J. 49 (2001), no. 1, 23-37.

[39] Shen, Z.: $L^{p}$ estimates for Schrödinger operators with certain potentials. Ann. Inst. Fourier (Grenoble) 45 (1995), no. 2, 513-546.

[40] Song, L. And Yan, L.: Riesz transforms associated to Schrödinger operators on weighted Hardy spaces. J. Funct. Anal. 259 (2010), no. 6, 1466-1490.

[41] Stegenga, D. A.: Bounded Toeplitz operators on $H^{1}$ and applications of the duality between $H^{1}$ and the functions of bounded mean oscillation. Amer. J. Math. 98 (1976), no. 3, 573-589.

[42] Sun, Y. AND Su, W.: Interior $h^{1}$-estimates for second order elliptic equations with vanishing LMO coefficients. J. Funct. Anal. 234 (2006), no. 2, 235-260.

[43] TANG, L.: Weighted norm inequalities for commutators of Littlewood-Paley functions related to Schrödinger operators. ArXiv: 1109.0100, 2011.

[44] Tang, C. AND Bi, C.: The boundedness of commutator of Riesz transform associated with Schrödinger operators on a Hardy space. J. Funct. Spaces Appl. 7 (2009), no. 3, 241-250.

[45] YANG, D. AND YANG, D.: Characterizations of localized $\mathrm{BMO}\left(\mathbb{R}^{n}\right)$ via commutators of localized Riesz transforms and fractional integrals associated to Schrödinger operators. Collect. Math. 61 (2010), no. 1, 65-79.

[46] Yang, D. And YAnG, S.: Local Hardy spaces of Musielak-Orlicz type and their applications. Sci. China Math. 55 (2012), no. 8, 1677-1720.

[47] Yang, D. And Zhou, Y.: Localized Hardy spaces $H^{1}$ related to admissible functions on RD-spaces and applications to Schrödinger operators. Trans. Amer. Math. Soc. 363 (2011), no. 3, 1197-1239.

[48] Zhong, J.: Harmonic analysis for some Schrödinger type operators. Ph.D. Thesis, Princeton University, 1993.

Received December 21, 2013.

LUONG DANG KY: Department of Mathematics, University of Quy Nhon, 170 An

Duong Vuong, Quy Nhon, Binh Dinh, Viet Nam.

E-mail: dangky@math.cnrs.fr 